\title{
VERFASSUNGSREGISTER AFRIKA
}

\author{
Von Brun-Otto Bryde und Hellmuth Hecker
}

\section{Vorbemerkung}

Nachdem der vierte und letzte Band (Afro-Asien) des vom Institut für Internationale Angelegenheiten herausgegebenen Verfassungsregisters 1963 erschien ${ }^{1}$, haben sich in den seither verstrichenen 12 Jahren so viele Änderungen auf dem Gebiete des Verfassungsrechts ergeben, daß eine Fortführung des Werkes dringend notwendig war. Es wurde beschlossen, diese Fortsetzung nach Kontinenten vorzunehmen und mit Afrika zu beginnen. Dafür sprechen zwei Gründe: Einmal gab es bei Afrika die meisten Änderungen und zum zweiten ist dieser Kontinent weitaus am besten dokumentiert, während Übersichten zur Gesetzgebung der anderen Kontinente so gut wie völlig fehlen. Keine einzige dieser Dokumentationen erlaubt jedoch einen vollständigen Überblick über die verfassungsrechtlich relevante Gesetzgebung. Das gleiche gilt für die für Afrika reich blühenden Verfassungssammlungen, die auf englisch (CAS), italienisch (Foderaro), französisch (Gonidec, Lavroff/Peiser, Muracciole) und russisch (KGA) vorliegen $^{2}$. Diese Sammlungen gingen - ebenso wie die universelle Sammlung v. Peaslee - immer noch von der Vorstellung des 19. Jahrhunderts aus, wonach die Verfassung eines Staates der ruhende Pol in den Erscheinungen (der Einzelgesetze) Flucht war. Heute dagegen ist die Verfassung das Unstabilste eines Staates in Afrika - bei jedem der nicht abreißenden Militärputsche und Revolutionen wird in der Regel als erstes die Verfassung außer Kraft gesetzt. Aber selbst verhältnismäßig stabile Staaten ändern und ergänzen ihre Verfassungen kontinuierlich, oft in wichtigen Fragen. Angesichts dessen wäre es verfehlt, heute noch eine gebundene afrikanische Verfassungssammlung herauszugeben, womit nicht gesagt sein soll, daß das Fehlen einer solchen Sammlung in deutscher Sprache allein diesen Vernunftgrund hätte. Die einzige Möglichkeit besteht heute in einer Loseblattausgabe in der Form, wie sie die universelle Sammlung von Blaustein/Flanz und die als Beilage zu dieser Zeitschrift erscheinenden „Verfassungstexte“ praktizieren, oder im Abdruck der jeweils neuen Verfassungen in Zeitschriften und Schriftenreihen.

Der häufige Wechsel der Verfassungen erschwert einen Überblick und ein Auffinden der Texte sehr. Daher erscheint es wesentlich sinnvoller, ein Register der Fundstellen aller Texte herauszugeben, als die bereits vielfach abgedruckten Texte noch einmal zu publizieren. Ein solcher Versuch wurde bisher nur einmal unternommen.

P. H. Karlsson, Sources for African Constitutional Studies, in: African Law Studies 1971 Nr. 5, S. 57-98 und 1972 Nr. 6, S. 123-168.

So nützlich dieser Versuch ist, so leidet er doch an Mängeln. Erstens umf́aßt er nur einen Teil der Staaten Afrikas, und zweitens ist er besonders für Ainderungs-

1 H. Hecker, Verfassungsregister, Teil IV: Afrika - Asien - Australien, Frankfurt/M. 1963.

2 Vgl. die UUbersicht von Rogge, VRU 1974, S. 207. 
gesetze unvollständig und unzuverlässig. Außerdem erfaßt er nur amtliche Fundstellen und nicht die, für den interessierten Wissenschaftler oft leichter zugänglichen, sonstigen Veröffentlichungen.

Die vorliegende Fortsetzung des Verfassungsregisters knüpft an den Hauptband an, bringt bei Anderung von Verfassungen oder weiteren Fundstellen den letzten im Register von 1963 genannten Text aber noch einmal. Der Begriff der Verfassung wird hierbei nicht $\mathrm{zu}$ eng und formell gezogen, so daß auch Statuten einer Militärregierung und dergl. materielle Verfassungsgesetze möglichst mit erfaßt werden.

Eine Schwierigkeit bei der Zusammenstellung des Registers lag darin, daß die Lieferungen der Gesetzesblätter, die das Hamburger Afrika-Institut mühevoll gesammelt hat, oft schleppend und lückenhaft erfolgen. Während grundsätzlich Vorschriften bis Ende 1974 aufgenommen sind, können die Verfasser nur bis Ende 1973 eine größtmögliche Vollständigkeit verbürgen. Da meist Jahresregister fehlen, ist eine absolute Kontrolle allerdings nicht möglich. Da die Beschaffung und Sichtung des Materials für einen Bearbeiter allein zu mühsam ist, teilten sich zwei Referenten des Instituts für Internationale Angelegenheiten in diese Arbeit. Dabei übernahm einer (Bryde) die anglophonen, der andere (Hecker) die frankophonen Staaten, während die übrigen zwischen beiden geteilt wurden.

Bei den frankophonen Staaten ist als amtliche Fundstelle Jahr und Seite der Journal Officiell zitiert. In den anglophonen Staaten werden Rechtsvorschriften als Supplement zu den Gazettes veröffentlicht, wobei besondere Reihen für Acts (bzw. Laws oder, z. B. bei Militärregierungen, Decrees) Subsidiary Legislation (L. N. oder G. N.) und Bills bestehen. Da diese in den meisten Bibliotheken nicht zusammen mit der Gazette, sondern getrennt und in der Reihenfolge der $\mathrm{Nu}$ merierung gebunden werden, ist auch hier lediglich Art der Rechtsvorschrift (A., D., G. N.) und Nummer zitiert.

Die Verfasser sind für jeden Hinweis, insbesondere auf weitere Fundstellen, dankbar. 


\section{Erlangung der Unabhängigkeit bestehender Staaten}

unvordenklich

26. 7.1847

31. 5.1910

28. 2. 1922

24. 2.1951

1. 1.1956

2. 3.1956

20. 3.1956

6. 3.1957

30. 9.1958

1. 1.1960

27. 4.1960

19. 6.1960

26. 6.1960

30. 6.1960

1. 7.1960

15. 7.1960

3. 8.1960

5. 8.1960

7. 8.1960

11. 8.1960

13. 8.1960

15. 8.1960

20. 8.1960

1. 10.1960

28. 11.1960

27. 4. 1961

9. 12. 1961

1. 7.1962

1. 7.1962

3. 7.1962

9. 10.1962

18. 12.1963

6. 7.1964

24. 10. 1964

18. 2.1965

11. 11.1965

30. 9.1966

4. 10.1966

12. 3.1968

6. 9.1968

12. 10.1968

10. 9.1974

25. 6. 1975

5. 7.1975

12. 7.1975
Äthiopien (Abessinien)

Liberia

Südafrika

Ägypten

Libyen

Sudan

Marokko

Tunesien

Ghana (Goldküste)

Guinea

Kamerun

Togo

Mali (-Föderation)(ehem. Franz. Sudan)

Madagaskar

Zaire (Kongo-Léopoldville)

Somalia

Gabun

Niger

Obervolta

Elfenbeinküste

Tschad

Zentralafrika (Ubangi-Chari)

Kongo (-Brazzaville)

Senegal

Nigeria

Mauretanien

Sierra Leone

Tanzania (Tanganyika u. Zanzibar)

Burundi (Urundi)

Rwanda

Algerien

Uganda

Kenia

Malawi (Nyassaland)

Zambia (Nord-Rhodesien)

Gambia

Rhodesien (Süd-Rhodesien), Unabhängigkeit nicht allgemeın anerkannt

Botswana (Betschuanaland)

Lesotho (Basutoland)

Mauritius

Swaziland

Äquatorialguinea (Span. Guinea)

Guinea-Bissao (Port. Guinea)

Mozambique

Kapverde

Sao Tomé e Principe 


\section{Spätere Erklärung zur Republik}

$\begin{aligned} \text { 18. 6. } 1953 & \text { Agypten } \\ \text { 25. 7.1957 } & \text { Tunesien } \\ \text { 1. 7. } 1960 & \text { Ghana } \\ \text { 31. 5. } 1961 & \text { Südafrika } \\ \text { 9. } 12.1962 & \text { Tanzania } \\ \text { 1. } 10.1963 & \text { Nigeria } \\ \text { 9. } 10.1963 & \text { Uganda } \\ \text { 12. } 12.1964 & \text { Kenia } \\ \text { 6. 7. } 1966 & \text { Malawi } \\ \text { 28. } 11.1966 & \text { Burundi } \\ \text { 1. 9. } 1969 & \text { Libyen } \\ \text { 2. 3. } 1970 & \text { Rhodesien } \\ \text { 24. 4. } 1970 & \text { Gambia } \\ \text { 19. 4. } 1971 & \text { Sierra Leone }\end{aligned}$

Unwirksame Unabhängigkeitserklärungen

11. 7. 1960

Katanga

31. 12. 1960

Buganda

30. 5.1967

Biafra

\section{Abkürzungen}

$\begin{array}{ll}\text { A } & \text { Act } \\ \text { ACR } & \text { Africa Contemporary Record (London), hrsg. v. C. Legum } \\ \text { ALD } & \text { African Law Digest } \\ \text { Aranzadi } & \text { Collección legislativa de España } \\ \text { ARB } & \text { Africa Research Bulletin, Exeter } \\ \text { BLE } & \text { Boletín de legislación extranjera } \\ \text { CA } & \text { Constituent Assembly Act } \\ \text { B. O. } & \text { Bulletin Officiel (Burundi, Marokko) } \\ \text { BOE } & \text { Boletín oficial del Estado (Spanien) } \\ \text { CAS } & \text { Constitutions of African States 2 Bde, New Delhi/New } \\ & \text { York 1972 } \\ \text { CCW } & \text { Constitutions of the Countries of the World, Blaustein/ } \\ \text { Corpus } & \text { Flanz (eds) } \\ \text { CPE } & \text { Corpus constitutionel, Leiden. Fasc. I 1968, II 1970, III 1972 } \\ \text { CP Inf. } & \text { Chronique de Politique Etrangére } \\ \text { D } & \text { Constitutional and parliamentary information (Genf) } \\ \text { DdG } & \text { Decree, Decreto, Decret } \\ \text { DLAA } & \text { Diário do Governo (Portugal) } \\ \text { Dumon } & \text { Documentation législative et administrative africaine } \\ & \text { La Communauté franco-afro-malgache, Brüssel 1960 }\end{array}$


EA

$\operatorname{EPC}(\quad)$

Foderaro

GA

Gaz.

GN

Gonidec

Hauptband

Inf. CP

Inf. jur.

J. O.

JÖR

KGA

$\mathrm{L}$

Lavroff (u. Peiser)

M. B.

MEJ

Muracciole

NE

$\mathrm{O}$

O. M.

$\mathrm{P}$

Peaslee

Perin

RDPSP

R. I.

RJP

Rev. égy

RTD

S. I.

State Papers

VA

VRƯ

YHR
Europa-Archiv

Encyclopedie Politique et Constitutionel, Paris, (Verfasser)

Le Costituzioni Africane, 3 Bde, Rom 1969

UN General Assembly

Gazette

Government (General) Notice

Constitutions des états de la communauté, Paris 1959

Verfassungsregister Teil IV: Afrika-Asien-Australien, Frankfurt/Main 1963

Informations Constitutionelles Parlamentaires (Genf)

Información jurídica (Madrid)

Journal Officiel

Jahrbuch des Öffentlichen Rechts

Konstitucii Gosudarstv Afrika, 3 Bde, Moskau 1963, 1966

Session Laws, Liberia

Les constitutions Africaines, 2 Bde, Paris 1964

Moniteur Belge

Middle East Journal

Les constitutions des états africaines d'expression française, Paris 1969

Notes et études (Paris)

Order

Oriente Moderno

Proclamation

Constitutions of Nations, 3. Aufl. Bd. 1, Africa, Haag 1965

Les institutions politiques du Congo indépendant au 30 Juin 1960, Léopoldville 1960

Revue de droit public et de la science Politique

Relazioni internazionale

Revue juridique et politique (Paris)

Revue égyptienne de droit international

Revue tunesienne de droit

Statutory Instrument (UK)

British and Foreign State Papers (London)

Volksabstimmung

Verfassung und Recht in Übersee (Hamburg)

Yearbook on Human Rights (New York)

\section{AGYPTEN}

Seit 1841 Vasallenstaat des Ottomanischen Reiches. 1882 von England besetzt, von 1914 bis 1922 britisches Protektorat. Am 18. 6. 1953 Republik. Vom 1. 2. 1958 bis 28. 9. 1961 mit Syrien zur „Vereinigten Arabischen Republik “ zusammengeschlossen. Dieser Name wurde nach Aufhebung der Vereinigung beibehalten, bis am 1. 9. 1971 wieder der Name Ägypten angenommen wurde.

Vorschriften bis 1958 (Provisorische Verfassung der VAR) im Hauptband S. 16. 
27. 9. 1962

Verfassungsproklamation

ARAB. TEXT: al ahram v. 28. 9. 1962

23. 3. 1964

Prov. Verfassung der VAR

in Kraft: 25. 3. 1964

ARAB. TEXT: al ahram v. 24. 3. 1964; Ad-Dustur 23. März 1964

(Die Verfassung vom 23. 3. 1964), Kairo o. J. (Informationsamt)

DT. ÜBERS.: Afrika heute v. 15. 4. 1964, Dok. Nr. 135

ENGL. UBERS.: Orient 1964, S. 33; V.A.R., The Constitution, Cairo 1964; CP Inf. 1964, S 112; Al-Marayati, S. 416

FRANZ. UBERS.: The Egyptian Directory 1963/4; RJP 1964, S. 319; Inf. CP 1964, S. 120; KDPS P. 1965, S. 925.

ITAL. UBERS.: OM 1964, S. 685; RI 1964, S. 727

SPAN. UBERS.: BLE 1965, S. 377

11. 9.1971 Verfassung der Arabischen Republik Ägypten

ARAB. TEXT: al ahram v. 11. 9. 1971

ENGL. ÜBERS.: Egyptian Gazette v. 10. 9. 1971; CCW (1972); VRU, Beilage „Verfassungstexte“, 1972; CPI 1974, S. 31; Peaslee", Africa, S. 152

FRANZ. UBBERS.: La Constitution permanente de la République; Arabe d'Egypte. Staatl. Informationsdienst Kairo 1971; RDPScP 1972, 1150; Inf. CP 1974, S. 35

\section{AQQUATORIAL-GUINEA}

Span. Guinea unabhängig von Spanien am 12. 10. 1968 als Republik ÄquatorialGuinea (

Vorschriften bis 1959 s. Hauptband S. 343.

20. 12. 1963 Gesetz Nr. 191: Grundlagen der Autonomie v. Äquatorialguinea in Kraft: 1. 1. 1964. Dadurch wird das Gesetz Nr. 46 v. 30. 7.1959 aufgehoben

AMTL. TEXT: BOE Nr. 312 v. 30.12. 1963

SPAN. TEXT: Aranzadi 1963, Nr. 2465

3. 7.1964 Dekret Nr. 1885: Genehmigung des ausführl. Autonomiegesetzes f. ӒG

AMTL. TEXT: BOE Nr. 161 v. 6. 7.1964

SPAN. TEXT: Aranzadi 1964, Nr. 1448

27 7.1968 Gesetz Nr. 49: Ermächtigung zum Gesetz der Unabhängigkeit f. ÄG

AMTL. TEXT: BOE Nr. 181 v. 29.7. 1968

SPAN. TEXT: Aranzadi 1968, Nr. 1327

16. 8.1968 Dekret Nr. 2070: Verfassung von ÄG

Die Verfassung war durch VA v. 11. 8. 1968 v. AG gebilligt worden

in Kraft: 12.10 .1968 - 3.8.1973

AMTL. TEXT: BOE Nr. 199 v. 19. 8. 1968 (Ber.: Nr. 221 v. 13. 9.) 
ENGL. UBERS.: YHR 1968, S. 119 (Auszug)

SPAN. TEXT: Cronica Nacional Nr. 275 v. 27. 7. 1968, Diario SP, S. 9; Inf. jur. Nr. 301 v. 1969, S. 59; Revista de política int. (Madrid) Nr. 99 v. 1968; Aranzadi 1968, Nr. 1505 (Ber.: Nr. 1591)

9. 10.1968 Dekret Nr. 2467: Gewährung der Unabhängigkeit für ÄG

AMTL. TEXT: BOE Nr. 245 v. 11. 10. 1968

SPAN. TEXT: Aranzadi 1968, Nr. 1784

4. 8.1973 Verfassung von AG

VA: 29.7. 1973

in Kraft: 4. 8.1973

Amtl. Text: BOE v. AG v. 4. 8. 1973

ENGL. UBERS.: UN, Office of Public Inf., Press Section, NV/354 v. 21. 12. 1973; CCW, Equitorial Guinea, 1974

\section{3. ÄTHIOPIEN}

Mit Ausnahme der italienischen Besetzung 1936-1942 immer unabhängig.

Die Provinz Eritrea war seit 1890 italienische Kolonie; 1941-1952 unter britischer Militärverwaltung; 1952-1962 autonomes Gebiet in Personalunion unter der Krone Aithiopiens. Am 15.11. 1962 wurde die Autonomie beseitigt.

Das amtliche Gesetzblatt Äthiopiens, die Negarit Gazeta, erscheint außer in der Amtssprache Amharisch auch in Englisch. Bei Divergenzer gilt der Amharische Text.

4.11.1955 Revidierte Verfassung des Kaiserreichs Äthiopien

s. Hauptband s. 31

dazu

Änderung: 21. 8. 1974 (P. 334/74, Neg. Gaz. 33 (1973/74), S. 132), ändert Art. 131

weitere Fundstellen

AMHARISCHER TEXT: Yetetekalelu hegotsch sera fitsame S. 1

ENGL. TEXT: Consolidated Laws of Ethiopia S. 1, CCW (1971); CAS Bd. 1, S. 262; Peaslee 4 , Africa, S. 182

FRANZ. UBBERS.: Revue Outre Mer 1970, S. 271; Inf. CP 1967, S. 1

ITAL. UBERS.: Foderaro Bd. 1, S. 229; Gatteschi, S. 506

RUSS. ÜBERS.: KGA 3, S. 681

12. 9.1974 Provisional Military Government Establishment Proclamation, suspendiert Verfassung von 1955 und richtet provisorisch Militärregierung ein

AMTL. TEXT

(AMHARISCH UND ENGLISCH): P. 1/1974, Neg. Gaz. 34 (1974/75), S. 1

ENGL. TEXT: Keesings 1974, 26733 (Auszüge) 
15. 9.1974 Definition of Powers of the Provisional Military Administration Council and its Chairman

AMTL. TEXT

(AMHARISCH UND ENGLISCH): P. 2/1974, Neg. Gaz. 34

Sondergebiet Eritrea

(1974/75), S. 3

15. 9.1974 Termination of the Federal Status of Eritrea

AMTL. TEXT

(AMHARISCH UND ENGLISCH): 0.27/1962, Neg. Gaz. 22 $(1962 / 63)$

\section{AFAR/ISSA}

Das TOM „Franz. Somaliküste“ hieß seit 1967 als TOM „Territorium des Afar/IssaVolkes". Frühere Vorschriften: Hauptband S. 283 ff. Dazu Penant 1968, S. 2-47; RJP 1974, S. 63-80.

30. 7.1963 Gesetz Nr. 759 betr. die Territorial-Versammlung v. Franz. Somaliküste

Änderungen:

1) 24. 10. 1968, Gesetz Nr. 916, ändert Tablau in Art. 2 (J. O. S. 10067)

2) 29. 12. 1972, Gesetz Nr. 1224, ändert Art. 2 (J. O. S. 13781)

AMTL. TEXT: J. O. v. 31. 7. 1963, S. 7077

22. 12. 1966 Gesetz Nr. 949 betr. Volksbefragung der Franz. Somaliküste

AMTL. TEXT: J. O. v. 23. 12. 1966, S. 11304

26. 1. 1967 Dekret Nr. 73 betr. deren Durchführung

AMTL. TEXT: J. O. v. 28. 1. 1967, S. 1011

3. 7.1967 Gesetz Nr. 521 betr. die Organisation des Territoriums der Afar/Issa

Änderung: 29. 12. 1972, Gesetz Nr. 1224 ändert Art. 2, 6, 25, 30 (J. O.S. 13781)

AMTL. TEXT: J. O. v. 4. 7. 1967, S. 6643 (Arrété Nr. 1379 v. 5.7.1967)

14. 2. 1968 Dekret Nr. 146 betr. Befugnisse des Hohen Kommissars AMTL. TEXT: J. O. v. 16. 2. 1968, S. 1723

21. 9.1972 Dekret Nr. 864 betr. Schaffung eines „groupement nomade autonome“

AMTL. TEXT: J. O. v. 25./26. 9. 1972, S. 10155

\section{ALGERIEN}

Unabhängig von Frankreich durch französische Erklärung v. 3. 7. 1962 ab diesem Tage.

10. 9. 1963 Verfassung der demokratischen und Volksrepublik Algerien Beschluß: 28. 8. 1963, Verkündung: 10.9.1963

VA: 8. 9. 1963 
AMTL. TEXT: J. O. v. 10. 9. 1963, S. 888 (Ber.: S. 1046)

ARAB. TEXT: Corpus I, S. 193

DT. ÜBERS. Afrika heute v. 15. 8. 1963, Dok. 132 (als Entwurf v. 31.7. 1963)

ENGL. ỦBERS. Peaslee3, Africa, S. 6; CAS S. 18; MEJ 1963, S. 446 (fehlerhaft); Middle East Forum Dez. 1963; CCW Algeria, 1972

FRANZ. TEXT: Inf. CP 1964, S. 2; NE Nr. 3063 v. 13. 2. 1964, S. 3; RDP 1964, S. 380; Annuaire Afrique du Nord 1963, S. 852;

Corpus Bd. I, S. 193; JOR 1974 (Bd. 23), S. 328

ITAL. UBERS.: RI 1964, S. 301; Foderaro Bd. 1, S. 1; O. M. 1963, S. 651

RUSS. ÜBERS.: KGA Bd. 2, S. 15

SPAN. ÜBERS.: Inf. jur. Nr. 254/5 v. 1964, S. 3

10. 7.1965 Ordonnance Nr. 182 betr. Bildung der Regierung (Revolutionsrat)

AMTL. TEXT: J.O. v. 13. 7. 1965, S. 671

FRANZ. TEXT: JÖR 1974 (Bd. 23), S. 334

\section{ANGOLA}

Über die portugies. Úberseeprovinz bis 1955 s. Hauptband S. 333. Unabhängigkeit für 11. 11. 1975 vorgesehen.

22. 11.1963 Dekret Nr. 45374 betr. (2.) Statut für Angola

AMTL. TEXT: DdG Nr. 274, S. 1784

22. 12. 1972 Dekret Nr. 45374 betr. (3.) Statut für Angola in $\mathrm{Kraft}: 1.1 .1973$

AMTL. TEXT: DdG Nr. 296, Suppl. v. 22. 12. 1972, S. 2030 (23)

11. 6.1974

Dekret Nr. 247 betr. Staatssekretäre für Angola

Änderung: 14. 9. 1974, Dekret Nr. 460 (DdG Nr. 215, S. 1093) AMTL. TEXT: DdG Nr. 135, S. 697

24. 7.1974 Gesetz Nr. 6 betr. Regierungs-Junten für Angola und Mozambique

AMTL. TEXT: DdG Nr. 171, Suppl., S. 846 (1)

27.11.1974 Gesetz Nr. 11 betr. Regierung des Staates Angola (Hoher Kommissar)

AMTL. TEXT: DdG Nr. 276, Suppl. v. 27. 11. 1974, S. 1464 (1)

15. 9. 1975 Vertrag von Portimao über die Unabhängigkeit Angolas

DT. ÜBERS.: EA 1975, D 293

ENGL. UBERS.: Africa Research Bulletin 1975, S. 3501 


\section{BOTSWANA}

Das ehemalige britische Protektorat Betschuanaland wurde am 30. 9. 1966 als Republik Botswana selbständig.

Vorschriften bis 1960, Hauptband S. 302.

21. 12. 1960 Bechuanaland Protectorate (Constitution) Order i. C.

Anderungen:

1) 27.9 .1963 (S. I. 1628, G. N. 90/63)

2) 27.7 .1964 (S. I. 1189, G. N. 121/64)

29. 1.1965 Bechuanaland Protectorate (Constitution) Order i. C.

Anderungen:

1) 18. 9. 65 (S. I. 1718)

AMTL. TEXT: S. I. 134

ENGL. TEXT: YHR 65, 375

20. 9.1966 Botswana Independence Order Constitution of Botswana

(Schedule 2)

in Kraft: 30.10. 1966

Anderungen:

1) 18. 8. 1969 (A. 30/69) ändert S. 5, 8, 13, 15, 25, 26, 110, $111,114,118,124,127$, hebt auf 23 ,

2) 22. 8. 1969 (A. 43/69) ändert S. 12, 33 und 37

3) 2. 7. 1970 (A. 25/70) ändert S. 9, 25, 26, 110, 111, 112, 113,114

4) 30. 10. 1972 (A. 28/72) ändert S. 59

5) 27.11 .1973 (A. 24/73) ändert S. 35, 59

AMTL. TEXT: S. I. 1171, LN 83/66

ENGL. TEXT: CCW (einschl. Änderung 1) und 3), 1972); CAS (ohne Änderung); Peaslee 4 , Africa, S. 11 (Fass. 1970)

FRANZ. TEXT: Corpus I, 145

\section{BRITISH INDIAN OCEAN TERRITORY}

Das British Indian Ocean Territory wurde 1965 als Kolonie aus den bisher zu den Seychellen gehörenden Farquhar und Aldabra Inseln und der bisher zu Mauritius gehörenden Chagos Inselgruppen gebildet.

8. 11. 1965 The British Indian Ocean Territory Order

Anderungen:

1) 26. 1. 1968 (S. I. 111) Korrigiert die Benennung der Inselgruppen

8.11.1965 Royal Instructions

AMTL. TEXT: S. I. 1920

AMTL. TEXT: S. I. 1965 Bd. 3, S. 6440 


\section{BURUNDI}

Unabhängig von Belgien am 1. 7. 1962, Republik am 28. 11. 1966.

23.11.1961 Vorläufige Verfassung des Königreichs Burundi,

Ordonnance législative Nr. 01/20

in Kraft: 30.1962

Änderung: 30. 6. 1962, Ord. legisl. betr. Aufhebung der Einschränkung des Inkrafttretens eines Artikels der Verfassung (B. O. Ruanda-Urundi 1962, S. 87)

AMTL. TEXT: B. O. Ruanda-Urundi Nr. 2 bis v. 6. 2. 1962, S. 67; B. O. Burundi 1962, Nr. 1, S. 1

DT. TEXT: Afrika Inf.-Dienst v. 15.7. 1962, Dok. 118

16. 10.1962 Verfassung des Königreichs Burundi

in Kraft: $1.7 .1962-8.7 .1966$

AMTL. TEXT: B. O. Nr. 1 bis 1. 1. 1963, S. 1

ENGL. UBBERS.: CP Inf. 1965, S. 66; Peaslee ${ }^{3}$, Africa, S. 19

FRANZ. TEXT: Inf. CP 1965, S. 66; NE Nr. 3175 v. 26. 3. 1965, S. 8; CPE 1963, S. 633; Corpus II, S. 51

ITAL. UBBERS.: Foderaro Bd. 1, S. 91

RUSS. UBBERS.: KGA Bd. 2, S. 40

SPAN. UBERS.: Inf. jur. Nr. 272/3 v. 1966, S. 23

8. 7.1966 Arrêté royal Nr. 001/2 betr. Aufhebung der Verfassung v. 1962

AMTL. TEXT: B.O. Nr. 9 v. 1. 9. 1966, S. 315

28.11.1966 Acte de proclamation: Ausrufung der Republik und Proklamation eines nat. Rev.komitees

AMTL. TEXT: B. O. Nr. 1 v. 1967, S. 1

FRANZ. TEXT: Corpus II, S. 57

19. 12. 1966 Dekretgesetz Nr. 1/6: Organisation der gesetzgebenden und reglementären Befugnisse

AMTL. TEXT: B. O. Nr. 1 v. 1967, S. 4

FRANZ. TEXT: Corpus II, S. 58

25. 1.1968 Dekretgesetz Nr. 1/133: Auflösung des nat. Rev.komitees

AMTL. TEXT: B. O. Nr. 3 v. 1968, S. 127

20. 10.1971 Dekretgesetz Nr. 1/144: Schaffung eines Obersten Rates der Republik

AMTL. TEXT: B. O. 1971, S. 471

FRANZ. TEXT: Corpus II, S. 58

11. 7.1974 Verfassung der Republik Burundi

AMTL. TEXT: B. O. Nr. 8 v. 1. 8. 1974, S. 211 


\section{DAHOMEY}

Unabhängigkeit v. Frankreich am 1. 8. 1960.

15. 2. 1959

25. 11.1960

28. 10. 1963

19. 12.1963

8. 4. 1968

26. 12.1969

7. 5.1970

26. 7.1972
(Erste) Verfassung der Republik Dahomey (in der Communauté) siehe Hauptband S. 70

(Zweite) Verfassung der Republik Dahomey, Gesetz Nr. 36

aufgehoben durch Ordonnance Nr. 1 v. 28. 10. 1963 (s. u.)

AMTL. TEXT: J. O. Nr. 30 v. 26. 11. 1960, S. 733

FRANZ. TEXT: NE Nr. 2994 v. 27. 5. 1963, S. 39; Lavroff, S. 75; RJP 1961, S. 262; Muracciole, S. 39

SPAN. UBBRS.: Inf. jur. Nr. 254/55 v. 1964, S. 35

RUSS. UBBERS.: KGA Bd. 1, S. 163

Ordonnance Nr. 1: Auflösung der nationalen Institutionen und Errichtung einer prov. Regierung

Änderung: 4. 12. 1963, Ord. Nr. 17, ändert Art. 3 (J. O. v. 23. 12. 1963, S. 11)

AMTL. TEXT: J. O. v. 23. 12. 1963, S. 2

(Dritte) Verfassung der Republik Dahomey, Ordonnance Nr. 8

Unterzeichnung: 19. 12. 1963; VA: 5. 1. 1964, Verkündung

11. 1.1964

AMTL. TEXT: J. O. v. 12. 1. 1964, S. 31

ENGL. ÜBERS.: CP Inf. 1964, S. 133; Peaslee ${ }^{3}$, Africa, S. 151

FRANZ. TEXT: RJP 1964, S. 161; Inf. CP 1964, S. 142; NE

Nr. 3175 v. 26.3 .1965 , S. $42 ; 3307$ v. 8.7 .1966 , S. 34

ITAL. ÜBERS.: Foderaro Bd. 1, S. 193

SPAN. UBBRS.: Inf. jur. Nr. 272/73 v. 1966, S. 3

(Vierte) Verfassung der Republik Dahomey, Ordonnance Nr. 20

VA: 31. 3. 1968; i. K. s. Ord. 26 v. 16. 4. (J. O. v. 17. 4., S. 327)

AMTL. TEXT: J. O. Nr. 9 v. 15. 4. 1968, S. 315

ENGL. UBERS.: YHR 1968, S. 203 (Auszug); CP Inf. 1969, S. 135

FRANZ. TEXT: RJP 1968, S. 923; Inf. CP 1969, S. 135; NE Nr. 3625 v. 2. 10.1969

Ordonnance Nr. 53: Charta des Direktoriums als vorl. Grundgesetz

AMTL. TEXT: J. O. v. 27. 12. 1969, S. 885

Ordonnance Nr. 34: Charta des Präsidentschaftsrates

AMTL. TEXT: J. O. Nr. 16 v. 1.7.1970, S. 450

ENGL. ÜBERS.: YHR 1970, S. 65 (Auszug); CCW, Dahomey, 1971; Peaslee ${ }^{4}$, Africa, S. 138

FRANZ. TEXT: RJP 1970, S. 517

ITAL. ÜBERS.: Foderaro Bd. 1, S. 222

Verfassung außer Kraft durch Staatsstreich (ARC 1972/3, C 122) 


\section{ELFENBEINKÜSTE}

Unabhängig v. Frankreich am 7. 8. 1960.

26. 3. 1959

Verfassung der Republik (in der Communauté)

siehe Hauptband S. 72, mit Änderung v. 27. 7. 1960

31. 10.1960

Verfassung der Republik, Gesetz Nr. 356

Änderung:

1) 11. 1. 1963, Gesetz Nr. 1, ändert Art. 64 (J. O. v. 14. 1., S. 29)

AMTL. TEXT: J. O. Nr. 58 v. 4. 11. 1960, S. 1271 (Ber.: Nr. 61 v. 18. 11. 1960)

ENGL. UBBERS.: Peaslee ${ }^{3}$, Africa S. 242; CAS S. 590; CCW, Ivory Coast, 1973; Peaslee ${ }^{4}$, Africa, S. 323

FRANZ. ÜBERS.: Lavroff, S. 61; RJP 1961, S. 255; Muracciole, S. 31; NE Nr. 2994 v. 27. 5. 1963, S. 39

ITAL. ÜBERS.: Foderaro Bd. 1, S. 181

RUSS. ÜBERS.: KGA Bd. 1, S. 27

SPAN. ÜBERS.: Inf. jur. Nr. 254/55 v. 1964, S. 51 (Fass. 1963)

\section{GABUN}

Unabhängigkeit von Frankreich am 15. 7. 1960.

19. 2.1959 (Erste) Verfassung der Republik (in der Communauté)

s. Hauptband, S. 74

9. 8. 1960 Gesetz Nr. 57 betr. Premierminister als vorläufigen Staatschef

AMTL. TEXT: J. O. der Communauté 1960, S. 131

14. 11.1960

(Zweite) Verfassung der Republik, Verfassungsgesetz Nr。68

s. Hauptband, S. 74

21. 2. 1961

(Dritte) Verfassung der Republik, Gesetz Nr. 1

Änderungen:

1) 31. 5. 1963, Gesetz Nr. 23, ändert Art. 63 (J. O. v. 1.7., S. 512)

2) 30. 6. 1966, Gesetz Nr. 17, ändert Art. 20 (J. O. v. 1. 7. 1966, S. 565)

3) 17. 2. 1967, Gesetz Nr. 1, ändert Art. 6-11, 21, 26-27 (J. O. v. 1. 3., S. 161)

4) 30. 3. 1967, Gesetz Nr. 4, ändert Art. 11 (J. O. v. 15. 4., S. 263)

5) 16. 6. 1967, Gesetz Nr. 16, ändert Art. 7 (J. O. v. 15. 7., S. 487)

6) 13. 12. 1967, Gesetz Nr. 21, ändert Art. 10, 21 (J. O. v. 15. 1. 1968, S. 25)

7) 29. 5. 1968, Gesetz Nr. 2 hebt auf Art. 4 (J. O. v. 1. 7., S. 403)

8) 9. 11. 1968, Gesetz Nr. 13, ändert Art. 6-11, 17 (J. O. v. 15. 12., S. 774)

9) 1. 6. 1969, Gesetz Nr. 1, ändert Art. 20, 23, 61 (J. O. v. 1. 7., S. 525) 
10) 29. 7. 1972, Gesetz Nr. 14, setzt Art. 4 in geänderter Fassung wieder in Kraft, ändert Art. 8-10 (J. O., SonderNr. v. 20. 12., S. 1)

AMTL. TEXT: J. O. Nr. 6 v. 25. 2. 1961, S. 147

DT. ÜBERS.: Afrika Inf.dienst. v. 21. 3. 1961, Dok. 84

ENGL. UBERS.: Peaslee ${ }^{3}$, Africa, S. 194; CP Inf. 1966, S. 2 (1963); CAS, S. 294 (nur Änderung Nr. 3 berücksichtigt!); Peaslee, 4 Africa, S. 206 (Fass. 1968)

FRANZ. TEXT: NE Nr. 2795 v. 10. 7. 1961, S. 47; 2994 v. 27. 5. 1963 , S. $52 ; 3175$ v. 26. 3. 1965, S. 24 (Änderung Nr. 1); Lavroff, S. 116; Muracciole, S. 59; RJP 1961, S. 284; Inf. CP 1966, S. 15 (Fass. 1963)

ITAL. UBBERS.: Foderaro Bd. 1, S. 251 (Fass. 1963)

RUSS. ÜBERS.: KGA Bd. 1, S. 78

SPAN. UBERS.: Inf. jur. Nr. 254/5 v. 1964, S. 15

\section{GAMBIA}

Unabhängig von Großbritannien a m 18. 2. 1965, Republik 24. 4. 1970.

Vorschriften bis 1962, Hauptband S. 305.

18. 4. 1962 The Gambia (Constitution) Order in Council

Anderungen:

1) 24. 8. 1962 (S. I. Nr. 1871)

2) 27.9 .1963 (S. I. Nr. 1629)

3) 27.7.1964 (S. I. Nr. 1190)

AMTL. TEXT: S. I. Nr. 826 (Bd. 2, S. 876), LN. 35/62

29. 1.1965 The Gambia Independence Order in Council

in $\mathrm{Kraft}:$ 18. 2. 1965

Schedule 3 enthält die Verfassung

Anderungen:

1) 8. 2.1966 (A. 3/66, ändert S. 1 [1])

2) 8. 2.1966 (A. 4/66, ändert S. 36 [2] a)

3) 15. 4. 1966 (A. 9/66, ändert S. 58)

4) 15. 4.1966 (A. 11/66, ändert S. 36)

5) 6. 9.1968 (A. 13/68, ändert S. 33 (1) c)

6) 10.7 .1969 (A. 10/69, ändert S. 36)

AMTL. TEXT: S. I. Nr. 135; LN 9/65; Laws of the Gambia 1966, Bd. 1, S. 11

ENGL. TEXT: CAS, I S. 324 (einschließlich Änd. 6)

ITAL. ÜBERS.: Foderaro I, S. 267 (Fass. 1965)

24. 4.1970 Verfassung der Republik Gambia. Constitution of the Republic of Gambia

1) 5. 6. 1972 (A. 12/72 ändert S. 66)

2) 29. 3.1973 (A. $1 / 73$ ändert S. 43 , neue S. 65 A)

AMTL. TEXT: A. $1 / 70$

ENGL. TEXT: CCW 1971 (ohne Änderungen); Peaslee 4 , Africa, S. 226 


\section{GHANA}

Die Goldküste unabhängig von Großbritannien am 6. 3. 1957, Republik am 1.7. 1960.

Vorschriften bis 1960 Hauptband, S. 75.

29. 6.1960 Verfassung der Republik

in Kraft: 1. 7. 1960

Änderungen:

1) 26. 1. 1964 (A. 224/64) ändert Art. 1, 2, 3, 7, 18, 20, 45 und 46

2) 20. 5. 1965 (A 290/65) ändert Art. 11

AMTL. TEXT: C. A. $1 / 60$

ENGL. TEXT: C. P. Inf. 1960, S. 118, 1961, S. 74

FRANZ. ÜBERS.: Inf. C. P. 1960, S. 122, 1961, S. 47; Lavroff/ Peiser II, S. 108; NE 2709

DS. ÜBERS.: Afrika Informationsdienst v. 1. 4. 1960 (Entwurf) ITAL. ÜBERS.: Foderaro I, S. 345

SPAN. ÜBERS.: Inf. Jur. Nr. 210/1, Nov./Dez. 1960, S. 33

RUSS. ÜBERS.: KGA Bd. 1, S. 113

24. 2.1966 Proclamation for the Constitution of a National Liberation Council

hebt Verfassung von 1960 auf, Staatsgewalt liegt beim Liberation Council

Anderungen:

1) 3. 5.1966 (NLCD 1/66)

2) 15. $11.1966(\mathrm{NLCD} 104 / 66)$

3) 8.41969 (NLCD 341/68)

AMTL. TEXT: Gaz. No. 11 v. 28. 2. 1966

18. 8.1966 National Liberation Council (Consequential and Transitory Provisions) Decree, 1966

Provisorische Verfassung

Änderungen:

1) 25. 4. 1969 (NLCD 349/68) ändert S. 15

AMTL. TEXT: NLCD 73/1966

15.11.1966 Constitutional Commission Decree

setzt Kommission zur Vorbereitung einer Verfassung ein

Änderungen:

1) 10. 3. 1967 (NLCD 149/1967)

2) 10. 4. 1967 (NLCD 158/1967)

3) 29. 11. 1968 (NLCD 311/1968)

AMTL. TEXT: NLCD 102/1966

10. 1.1968 Constituent Assembly Decree

AMTL. TEXT: NLCD 222/1968

5. 11.1968 Constituent Assembly (Nr. 2) Decree

(ersetzt NLCD 222/1968 v. 10. 1. 1968)

Änderungen:

1) 20.11. 1968 (NLCD 304/68)

2) 18. 8. 1969 (NLCD 380/69)

AMTL. TEXT: NLCD 299/68 
30. 10. 1969

22. 8.1969

13. 1. 1972

14. 4.1972
Constitution (Consequential and Transitional Provisions) Decree löst NLC auf und regelt Úbergabe der Macht an Zivilregierung AMTL. TEXT: NLCD 406/69

\section{Constitution of Ghana}

AMTL. TEXT: Constitution of the Republic of Ghana, Ghana Publishing Corporation, Accra-Tema

ENGL. TEXT: CAS, S. 435; YHR 1969, S. 79

National Redemption Council (Establishment) Proclamation

Suspendiert Verfassung, setzt NRC ein und überträgt ihm die Staatsgewalt

Änderungen:

1) 17. 1. 1972 (NRCD 1/72 ändert Zus. des Council)

2) 28. 3. 1972 (NRCD 49/72, ändert S. 16, 17)

3) 14. 4. 1972 (NRCD 58/72 ändert S. 24, hebt S. 25 auf)

AMTL. TEXT: Gazette v. 14. 1. 1972

ENGL. TEXT: CCW (1973)

\section{Affairs of State Decree}

regelt Führung der Staatsgeschäfte durch NRC

AMTL. TEXT: NRCD 58/72

ENGL. TEXT: CCW (1973)

\section{GUINEA}

Unabhängig von Frankreich durch Proklamation v. 2. 10. 1958 ab 30. 9. 1958.

10. 10. 1958

Verfassung der Republik Guinea, Gesetz Nr. 4

Änderung:

1) 31. 10. 1963, ändert Art. 2, 3, 7, 8, 11, 12, 16, 18, 34

(J. O. v. 15. 11., S. 282)

AMTL. TEXT: J. O. v. 12. 11. 1958, S. 87 (verkündet durch VO Nr. 15 v. 12. 11. 1958)

DT. UBERS.: Afrika Inf.dienst 1958, Dok. 30

ENGL. UBERS.: CP Inf. 1961, S. 81; State Papers Bd. 163, S. 917 (m. Unabh. Proklamation); YHR 1958, S. 90 (Auszug); Peaslee $^{3}$, Africa, S. 232; CAS, S. 575; Peaslee 4 , Africa, S. 313

FRANZ. TEXT: Gonidec, S. 52; Lavroff, S. 133; Muracciole, S. 71; Inf. CP. 1961, S. 111; RJP 1961, S. 295; NE Nr 2995 v. 30. 5. 1963; JÖR 1970, S. 533

ITAL. UBERS.: Foderaro, Bd. 1, S. 363

RUSS. UBERS.: KGA Bd. 1, S 146

SPAN. UBBER.: BLE 1961, S. 191 


\section{GUINEA-BISSAO}

Die portugies. Úberseeprovinz Guinea wurde am 10. 9. 1974 unabhängig, nachdem schon am 24. 9. 1973 im Osten des Landes eine Unabhängigkeitserklärung der Gegner Portugals erlassen worden war.

S. Hauptband S. 338 bis 1955.

22. 11. 1963 Dekret Nr. 45372 betr. das (2.) Statut für Guinea

AMTL. TEXT: DdG Nr. 274, S. 1772

22. 12. 1972 Dekret Nr. 542 betr. das (3.) Statut für Guinea

AMTL. TEXT: DdG Nr. 296, Suppl. v. 22. 12. 1972, S. 2030 (8)

24. 9.1973 Verfassung von Guinea-Bissao

mit Unabhängigkeitserklärung

ENGL. UBBERS.: UN, Gen. Ass. A 1196 v. 15. 10. 1973; CCW, Guinea-Bissau, 1974, ACR 1973/4, C 95

26. 8.1974 Übereinkommen Portugals mit der PAIGC Guineas über die Unabhängigkeit

Bekanntgemacht durch Erklärung in Algier am 26. 8. 1974

DT. ÜBERS.: EA 1974, D 428

ENGL. UBERS.: ILM 1974, S. 1244

FRANZ. TEXT: Le Monde Nr. 9212 v. 28. 8. 1974, RGDIP 1974, S. 1252

\section{KAMERUN}

Unabhängig von Frankreich (Treuhandmacht) am 1. 1. 1960.

Südkamerun (brit. Treuhandgebiet) angegliedert am 1. 10. 1961.

Bundesrepublik 1961-1972, seitdem Einheitsstaat.

4. 3.1960 Verfassung der Republik Kamerun

s. Hauptband S. 134

dazu:

1. 9. 1961 Verfassung der Föderativen Republik Kamerun, Gesetz Nr. 24

in Kraft: 1. 10. 1961. Daneben blieb die Verfassung v. 1960 in Kraft!

Änderungen:

1) 10. 11. 1969, Gesetz Nr. 14, ändert Art. 10, 11, 15, 16, 24-39, 44 (J. O. v. 1. 12. 1969, Sonder-Nr. 102)

2) 4. 5. 1970, Gesetz Nr. 1, ändert Art. 9 (J. O., Sonder-Nr. 1 v. 1. 6., S. 14)

AMTL. TEXT: J. O. Nr. 1459 bis v. 30. 9. 1961, S. 1090

ENGL. UBERS.: CP Inf. 1962, S. 1, YHR 1961, S. 45 (Auszug)

Peaslee ${ }^{3}$, Africa, S. 34, CAS, S. 142, CCW, Federal Republic of Cameroon, 1972 (Fass. 1970) (Änd. Nr. 1-2 im Anhang)

FRANZ. TEXT: Inf. CP 1962, S. 1, RJP 1961, S. 610

NE Nr. 2994 v. 27. 5. 1963, S. 12 Lavroff, Suppl. S. 6, CCW a. a. O.

ITAL. ÜBERS.: Foderaro Bd. 1, S. 105

SPAN. UBERS.: Inf. jur. Nr. $248 / 9$ v. 1964 , S. 41

RUSS. UBERS.: KGA Bd. 1, S. 195 
2. 6.1972 Verfassung des Einheitsstaates Republik Kamerun, Dekret Nr. 270

AMTL. TEXT: J. O. Nr. 4, Suppl. v. 2. 6. 1972, S. 32

ENGL. TEXT: Verfassungstext, Beilage zu VRU Nr. 1/74

CCW, United Rep. of Cameroon, 1973; ACR 1972/3, C 116;

Peaslee ${ }^{4}$, Africa, S. 86

FRANZ. TEXT: RJP 1973, S. 30

\section{ANHANG: SONDERGEBIETE}

\subsubsection{Verfassung des Gliederstaates Ostkamerun: Gesetz Nr. 1 \\ 1.11.1961 AMTL. TEXT: J. O. (Ostkamerun!) v. 1.11.1961}

FRANZ. TEXT: Cauris, Revue d'étude des institutions camerounaise et comparées, H. 4/1973, S. 233, Lavroff, Suppl., S. 18

26. 10. 1961

Verfassung des Gliedstaates Westkamerun (Südkamerun),

Gesetz Nr. 61

AMTL. TEXT: J. O. (Cameroun) Nr. 2 v. 13. 11.1961

FRANZ. TEXT: Lavroff, Suppl. S. 23

NE Nr. 2946 v. 17. 12. 1962, S. 20

Nr. 2994 v. 27. 5. 1963 , S. 18

SPAN. UBERS.: Inf. jur. Nr. 248/9 v. 1964, S. 55

27. 9. 1961 Notenwechsel Großbritanniens mit Kamerun betr. die Rückgabe Südkameruns

FRANZ. TEXT: State Papers Bd. 166, S. 102

\section{KAPVERDE}

Portugies. Úberseeprovinz, die einzige, die 1955 kein Statut erhielt. Unabhängig am 5. 7. 1975.

Vorschriften bis 1935 s. Hauptband S. 334.

22. 11. 1963 Dekret Nr. 45371: Statut für Kapverde

AMTL. TEXT: DdG Nr. 274 v. 1963, S. 1763

22. 12. 1972 Dekret Nr. 541: Statut für Kapverde

AMTL. TEXT: DdG Nr. 296, Suppl. v. 22. 12. 1972, S. 2030 (1)

21. 9. 1974 Übereinkommen Portugals mit der PAIGC über die

Unabhängigkeit

15. 11.1974 Gesetz Nr. 10 betr. einen Hohen Kommissar

AMTL. TEXT: DdG Nr. 266, Suppl. v. 15. 11. 74, S. 1388 (1)

\section{KENIA}

Unabhängig von Großbritannien am 12. 12. 1963, Republik 12. 12. 1964.

Vorschriften vor 1958 im Hauptband, S. 309 f.

3. 4. 1958 The Kenya (Constitution) Order in Council

Anderungen:

1) 28. 7.1959 (S. I. 1302)

2) 8.4 .1960 (S. I. 703)

3) 30.11 .1960 (S. I 2201) 

4) 27.4 .1961 (S. I 835)
$\begin{array}{ll}\text { 5) } 24.10 .1961 & \text { (S. I. 2036) }\end{array}$
6) 27.11 .1961 (S. I 2273)
7) 28.11 .1962 (S. I 2519)
8) 26. 3.1963 (S. I 610)

AMTL. TEXT: S. I. 600

11. 4.1963

4. 12.1963

Kenia Gazette v. 5. 4. 1958, Leg. Suppl. Nr. 15

\section{Kenya Order in Council}

Änderungen:

1) 30. 5. 1963 S. I. 1047 , L. N. $316 / 63$

AMTL. TEXT: S. I. 791/63

L. N. 245/63

Kenya Independence Order in Council

Constitution of Kenya (Schedule)

Anderungen:

1) 23. 11. 1964 (A 28/64) erklärt Kenia zur Republik mit Wirkung vom 12. 12. 1964, ändert S. 31, 32, 33, 34, 37, 42, 43, $46,48,56$ (2), 59, 60, 64, 65, 68, 69, 72, 74, 75, 76, 77, 81, $82,83,84,85,87,88,89,90,121,126,128,137,138,140$, $152,157,162,163,166,167,186,183,189,190,191,192$, 193, 196, 209, 210, 211, 223-238, 244, 245, Schedule 1, Schedule 5; streicht 73, 78, 79, 90, Chap. VII, 122, 123, 125, $139,141-151,153,155,158-161,164-165,168-170$, 187, 193, 194, 197-207, 222-224, 243, 246, Schedule 2

2) 11. 12. 1964 (A 38/64), ändert S. 9, 19, 25, 26, 29, 30, 42, $43,44,51,61,71,98,99,103,105,107,108,110,112,114$ $117,172,173,184,189,195,196,202,208,209,210,211$, $215,218,219,220,231,240,241,242,244,247$, S. 7, 8 , streicht Chap. VIII, 2, 3, Schedule 3

3) 3. 6.1965 (A 14/65), ändert S. 25, 26, 29, 30, 60, 66, $71,72,86,88,89,90,96,101,102,103,111,113,162,171$, $173,175,176,182,184,185,188,191$, Chap. XII, Part. 4, 116, 117, 180, 181, 183, Chap. XII, Part. 3, Schedule 1, 4

4) 5. 4. 1966 (A 16/66), ändert S. 6, 24, 30, 41, 42, 56, 87, $121,124,209,247$

5) 30. 4. 1966 (A 17/66) neuer 42a (Mandatsverlust nach Parteiaustritt

6) 3. 6. 1966 (A 18/66), ändert S. 27, 29

7) 3. 1.1967 (A 40/67), schafft Zwei-Kammer-System ab, ändert S. 29, 33c, 34, 35, 36, 39, 40, 41, 42, 42 A, 43, 44, 45 (6), 47, 48, 49, 50, 51, 52, 54, 57, 59, 60-65, 186, 244, 247 , Schedule 5

8) 29. 3. 1967 (A 4/67), interpretiert S. 42a, siehe Nr. 5

9) 10. 4. 1968 (A 16/68), ändert S. $20,38,41,48,66,244$, 247, streicht 36, Chap. VI, XIV, Schedule 5 III, 7, 8, 11 
10) 10. 7.1968 (A 45/68), führt Volkswahl des Präsidenten ein, ändert 29, $33 \mathrm{~A}, 33 \mathrm{~B}, 33 \mathrm{D}, 33 \mathrm{E}, 37,38,39,40,41,42 \mathrm{~B}$, 45, 74, 184, 186, 247, Schedule 5, streicht Schedule 6

AMTL. TEXT: S. I. 1968/63, L. N. 718/63 (Gaz. Suppl. v. 10. 12.1963)

ENGL. TEXT: Peaslee ${ }^{3}$ I, S. 257 (ohne Änderungen)

FRANZ. ÜBERS.: Inf. C. Part. 1967, 118

ITAL. ỦBERS.: Foderaro, Bd. II, S. 1 (ohne Änderungen)

10. 4. 1969 Constitution of Kenya

Neuveröffentlichung und Änderungen (2. Schedule)

in Kraft: 18. 4. 1969

AMTL. TEXT: A 5/69, Gaz. Suppl. v. 18. 4. 1969

ENGL. TEXT YHR 1969, 141, CCW (1971), CAS Bd., S. 606;

Peaslee $^{4}$, Africa, S. 339

FRANZ. UBERS.: EPC (Horrut) 1972

\section{KOMOREN}

Siehe Hauptband S. 273; dazu noch Penant 1970, S. 281-299. Eine VA vom 22. 12. 1974 sprach sich für die Unabhängigkeit aus, nur Mayotte stimmte für Verbleiben bei Frankreich. Mehrere Verfassungsentwürfe wurden ausgearbeitet.

3. 1.1968 Gesetz Nr. 4 betr. Ainderung des Gesetzes v. 22.12. 1961

betr. Organisation der Komoren

ändert Präambel, Art. 1-3, 5, 6, 9, 13-17, 19, 20, 28, 29, 3133; fügt ein Art. 8 bis, 11 bis, 11 ter, 37 bis; hebt auf Art. 18, 2126, 28 (5), 34-36

AMTL. TEXT: J. O. Nr. 2 v. 4. 1. 1968, S. 112

12. 4. 1969 Dekret Nr. 334 betr. Änderung v. Art. 15 des Dekrets v. 24. 9. 1946 betr. Verwaltungs-Reorganisation der Komoren AMTL. TEXT: J. O. Nr. 89 v. 16. 4. 1969, S. 3740

23. 11. 1974 Gesetz Nr. 965 über die Volksbefragung AMTL. TEXT: J. O. Nr. 275 v. 24. 11. 74, S. 11771

\section{KONGO(-ERAZZAVILLE)}

Kongo-Brazzaville unabhängig von Frankreich am 15. 8. 1960.

$1958 / 59$

2. 3.1961

8. 12. 1963
Zwölf Verfassungsgesetze

s. Hauptband S. 140-141

Verfassung der Republik Kongo, Gesetz Nr. 22

AMTL. TEXT: J. O. v. 4. 3. 1961, S. 2

FRANZ. TEXT: Lavroff, S. 238, Muracciole, S. 21, RJP 1961,

S. 245 , NE Nr. 2994 v. 27. 5. 1963, S. 32

RUSS. UBERS.: KGA Bd. 1, S. 222

Verfassung der Republik Kongo

AMTL. TEXT: J. O. v. 31. 12. 1963, S. 1039

DT. UBBERS.: Afrika heute v. 15. 2. 1964, Dok. 133

ENGL. UBERS.: CP Inf. 1965, S. 174, Peaslee ${ }^{3}$, Africa, S. 85 
FRANZ. TEXT: NE Nr. 3175 v. 26. 3. 1965, S. 34, Inf. CP 1965, S. 174, RJP 1964, S. 143

ITAL. UBBERS.: Foderaro Bd. 1, S. 149

RUSS. UBERS.: KGA Bd. 2, S. 419

SPAN. UBERS.: Inf. jur. Nr. $282 / 3$ v. 1966, S. 47

5. 8. 1968 Acte Nr. 5 betr. Schaffung des nat. Revolutionsrates

AMTL. TEXT: J. O. v. 15. 8. 1968, S. 382

14. 8.1968

Acte Nr. 1: Reglement intérieur des nat. Revolutionsrates

AMTL. TEXT: J. O. 15. 8. 1968, S. 382

14. 8.1968 Grundgesetz des Revolutionsrates

nur 28 Artikel, ersetzt die Verfassung v. 1963, soweit diese hiervon abweicht.

AMTL. TEXT: J. O. Nr. 16 v. 15. 8. 1968, S. 383

ENGL. UBERS: YHR 1968, S. 91 (Auszug)

1. 1. 1969 Acte fondamental Nr. 1

AMTL. TEXT: J. O. Nr. 1 v. 1. 1. 1969, S. 3

31. 12. 1969 Verfassung der Volksrepublik Kongo, Ordonnance Nr. 40 in Kraft: 1. 1. 1970

AMTL. TEXT: J. O. Nr. 2 v. 15. 1. 1970, S. 23

DT. UBERS.: Staat u. Recht 1970, S. 439

ENGL. ÜBERS.: CAS, S. 196, CCW, Congo, 1971; Peaslee4, Africa, S. 126

FRANZ. TEXT: RJP 1970, S. 117

Afrique Contemporaine Nr. 47 v. 1970, S. 15

SPAN. ÜBERS.: BLE 1970, S. 145

12. 7.1973

Verfassung

VA: 24. 6. 1973

in Kraft: 14. 7.1973

FRANZ. TEXT: RJP 1974, S. 15, Année Africaine 1973, S. 21

\section{LESOTHO}

Unabhängig vom brit. Protektorat (Basutoland) am 4. 10.1966 als Königreich. Vorschriften bis 1959 Hauptband S. 302.

14. 9.1959 Basutoland (Constitution) Order in Council

Änderungen:

1) 21.9 .1060 (S. I. Bd. 3, S. 4104)

2) 12.5 .1964 (S. I. Bd. 2, S. 3118)

3) 27.7 .1964 (S. I. Bd. 2, S. 3127)

4) 29.1.1965 (S. I. Bd. 1, S. 2569 Laws of Basutoland 1965 p. 1)

AMTL. TEXT: S. I. Bd. 2, S. 3308; O. G. High Commission, Nr. 3133 v. 23. 10.1959

ENGL. TEXT: YHR 1959, 1961, S. 347 (Auszug)

29. 1. 1965 Basutoland Order 1965

Constitution of Basutoland (Schedule 2)

AMTL. TEXT: S. I. Bd. 1, S. 2571 
Änderungen:

1) 1969 (A. 14/69) ändert S. 99, hebt auf S. 90

AMTL. TEXT: S. I. 1172; Suppl. to Gazette v. 4. 11. 1966 (4/1966)

10. 2. 1970

ENGL. TEXT: CCW (1971); YHR 66, 240

\section{Lesotho Order}

Die Verfassung wurde am 30. 1. 1970 suspendiert. Suspension wurde am 14. 2. 1970 mit Order 2/1970 bestätigt. Der Lesotho Order überträgt dem Premierminister (Tona-Kholo) Exekutive und Legislative

Ainderungen:

1) 5. 6. 1970 (O. 28), ändert S. 4 und 10, Exekutive und Legislative wird vom Regenten "on advice" des Premierministers ausgeübt.

2) 16.10.1970 (O. 45), ändert S. 4, 5, 6, 7, 9, 10; der trad. Ausdruck Tona-Kholo wird durch Prime Minister ersetzt. 5 (2) neues Amt Dep. Prime Minister

3) 18.6 .1971 (O. 25/71) ersetzt "regent“ durch König i. S. 4, 6, 10.

AMTL. TEXT: O. 1/70

ENGL. TEXT: CCW (1971)

\section{LIBERIA}

Von privaten US-Gesellschaften zur Ansiedlung befreiter Negersklaven gegründete Kolonie. Seit 27. 7. 1847 als Republik unabhängig.

26. 7.1847 Verfassung der Republik Liberia s. Hauptband S. 153 dazu Änderungen:

Anderung 7 des Hauptbands ist Anderung 9

7) 1945 (L 1944-45, c. 6), ändert II, 2, 3

8) 1947 (L 1945-46, c. 3), ändert I, 11

9) 1949 (L 1948-49, c. 1), ändert III, 1

10) 1951 (L 1950-51, c. 6), ändert II, 2, 5

11) 1955 (L 1954-55), ändert III, 1, 5

12) 1972 (L 1971-72), ändert I, 11; III, 3, 4; V, 5

Weitere Fundstellen

AMTL. TEXT: Liberian Code of Laws 1956 (einschließlich Änderung 11)

ENGL. TEXT: CAS Bd. 1, S. 861; CCW (1971); Peaslee ${ }^{3}$ Bd. 1, S. 422; Peaslee ${ }^{4}$, Africa, S. 418 (Fass. 1955)

FRANZ. UBERS.: Lavroff/Peiser II, S. 142; ECP (Tixier)

ITAL. UBERS.: Foderaro II, S. 203

RUSS. UBBERS.: KGA Bd. 1, S. 325 


\section{LIBYEN}

Das heutige Libyen war bis 1912 türkisch. 1912-1943 italienisch. 1943-1951 britische Militärverwaltung für die getrennten Gebiete Tripolitanien und Cyrenaika. Unabhängig am 24. 12. 1951 als bundesstaatliche Monarchie. 1963 Einheitsstaat. Am 1. 9. 1969 wurde die Monarchie gestürzt und die Republik ausgerufen.

7.10. 1951

Verfassung des Königreichs Libyen

Hauptband S. 157. Dazu Ainderungen:

1) 8.12.1962 (Annuaire d' Afrique Nord, S. 944), ändert Art. $36,172,179,182,184$

2) 25 . 4. 1963 (Annuaire d'Afrique Nord 1963, S. 946), ändert Art. 2, 3, 4, 26, 40, 44, 45, 68, 71, 75, 84, 85, 90, 94, 96, 98, $100,101,102,103,107,109,125,126,130,172,198,200$

weitere Fundstellen:

FRANZ. UBBERS.: Annuaire d'Afrique du Nord 1963

11. 12. 1969 Verfassungsproklamation

ENGL. UBERS.: Libyan New Agency v. 13. 12. 1969; CAS Bd. 1, S. 878; CCW (1974); VRU 1970, S. 385; Annuaire d'Afrique du Nord 1969, S. 943; Peaslee ${ }^{4}$, Africa, S. 433

FRANZ. ÜBERS.: Maghreb 1970 No. 38

DEUTSCHE UBBERS.: Staat und Recht 1970, S. 1011

ITAL. UBERS.: OM 1969, S. 701

\section{MADAGASKAR}

Unabhängig von Frankreich am 26. 6. 1960.

29. 4. 1959 Verfassung der malegesischen Republik, Verfassungsgesetz Nr. 2 (zunächst innerhalb der Communauté)

Änderungen:

1) 28. 6. 1960, ändert Art. 11, 14, 46, 69 (J. O. Mad. v. 2. 7., S. 1115; J. O. Communauté 1960, S. 54)

2) 6. 6. 1962, Gesetz Nr. 7, ändert Präambel, Art. 1, 5, 8, 9, $11,15,21,22,27,30,32,34,39,40$; hebt auf Art. 41 und numeriert Art. 42 ff. um in Art. 41 ff.; ändert die Art. 46 und 54 neuer Zählung, hebt auf Art. 55-64, 66-70 neuer Zählung; Art. 72 alter Zählung wird jetzt Art. 56 (J. O. v. 16. 6., S. 1076)

3) 27. 12. 1962, Gesetz Nr. 33, ändert Präambel (J. O. v. 18. 1. 1963, S. 84)

4) 27. 12. 1962, Gesetz Nr. 36, ändert Art. 32 (J. O. v.

18. 1. 1963, S. 89)

5) 5. 7. 1966, Gesetz Nr. 12, ändert Art. 46 (J. O. v. 16. 7., S. 1517)

6) 13. 10. 1970 Gesetz Nr. 18, ändert Art. 7, 9, 10, 16, 17, 44

(J. O. v. 23. 10., S. 2455) 
AMTL. TEXT: J. O. Mad. v. 29. 4. 1959, S. 1097; J. O. Communauté v. 15. 6. 1959, S. 71 u. 15. 7. 1960, S. 54

ENGL. UBERS.: YHR 1959, S. 193 (Auszug); C. P. Inf. 1960, S. 25 ; 1965, S. 30 (Fass. 1962); CAS, S. 891; Peaslee ${ }^{3}$, Africa, S. 456 (Fass. 1962); Peaslee ${ }^{4}$, Africa, S. 444 (Fass. 1971); CCW, Malagasy Republic (1974)

FRANZ. TEXT: GONIDEC, S. 169; Lavroff, S. 143 (Änd. Nr. 2: Suppl., S. 37); Muracciole, S. 83; Dumon, S. 232; Inf. CP 1960, S. 26; 1965, S. 46 (Fass. 1962); NE Nr. 2737 v. 23. 12. 1960 , S. $52 ; 2945$ v. 30. 12. 1963 , S. 13

ITAL. UBERS.: Foderaro Bd. 2, S. 235

RUSS. ÜBERS.: KGA Bd. 1, S. 443 (Fass. 1962)

SPAN. ÜBERS.: Inf. jur. Nr. $266 / 7$ v. 1965, S. 25 (Fass. 1962)

7.11. 1972 Verfassungsgesetz, Dekret Nr. 239

Art. 8 hält die Verfassung v. 1959 als einfaches Gesetz aufrecht, soweit sie nicht diesem Verfassungsgesetz widerspricht.

AMTL. TEXT: J. O. v. 8. 11. 1972, S. 2991

ENGL. UBERS.: Peaslee ${ }^{4}$, Africa, S. 442; CCW a. a. O., S. 14

26. 12.1972

Ordonnance Nr. 49 betr. Übertragung der Befugnisse des Staatspräsidenten auf den Regierungschef

AMTL. TEXT: J. O. v. 30. 12. 1972, S. 3512

ENGL. ÜBERS.: CCW, a. a. O., S. 13

\section{MALGACHISCHE INSELN}

s. Hauptband S. 286. Ausführliche Darstellung der Rechtslage: Penant 1974, S. $136-170$.

1. 4. 1960 Dekret Nr. 55 betr. Unterstellung der 5 Inseln unter den Minister für TOM und DOM

AMTL. TEXT: J. O. v. 14. 6. 1960, S. 5343

FRANZ. TEXT: Penant 1960, S. 424

\section{MALAWI}

Das britische Protektorat Nyassaland wurde 1953 Teil der Föderation von Rhodesien und Nyassaland. Nach deren Auflösung (1963) wurde es am 6. 7. 1964 unter dem Namen Malawi unabhängig und am 6. 7. 1966 Republik

26. 6. 1961 Nyasaland (Constitution) Order in Council

Hauptband S. 317

dazu Änderungen:

2. 5.1963 Nyasaland (Constitution) Order in Council

Anderungen:

1) 20.12 .1963 (S. I 2092)

2) 26. 3. 1964 (S. I. 493, G. N. 136/64)

AMTL. TEXT: S. I. 883 ; G. N. 97/63 
23. 6. 1964

14. 6.1966
Malawi Independence Order in Council. Constitution of Malawi (Schedule)

Änderungen:

1) 12. 11. 1964 (A 1/64), ändert S. 2, 13, 39, 46, 59, 61, 62, 70, 95, 96, 98, fügt S. 63 A ein

2) 1965 (A 41/65), ändert S. 80

3) 1965 (A 49/65)

AMTL. TEXT: S. I. 916/64; G. N. 215/65

ENGL. TEXT: Peaslee $^{3}$ I, S. 476 (ohne Änderung)

Republic of Malawi (Constitution) Act

(Verfassung ist 2. Schedule)

in Kraft: 6. 7. 1966

Änderungen:

1) 26. 8. 1966 (A 39/66), ändert S. 13, 19, 25, 26, 32, 67 und 72

2) 7. 2. 1968 (A 6/68), ändert S. 2, 11, 50, 68, 69

3) 28.10 .1968 (A 32/68), hebt S. 88 (2) auf

4) 24. 11.1969 (A. 25/69), ändert S. 19, 21, 31, 69, 70 und 98

5) 1. 4.1970 (A 1/70), ändert S. 63 (3)

6) 24. 8.1970 (A 19/70), ändert S. 20

7) 16.12.1970 (A 35/70), ändert S. 9 und 10

8) 6. 8. 1971 (A 25/71), ändert S. 19, 49, 50, hebt auf S. 27

9) 21. 3. 1972 (A 10/72) ändert S. 10, 3, 98

AMTL. TEXT: A 23/66; Laws of Malawi Rev. Ed. 1968

Vol. 1, Vor Chap. 1 (erfaßt Änderung 1)

ENGL. TEXT: CAS Bd. 1, S. 918 (einschl. Änderung 1) CCW

(1971); Peaslee 4 , Africa, S. 464 (Fass. 1971)

ITAL. UBERS.: Foderaro, Bd. II, S. 255

\section{MALI}

Die Mali-Föderation wurde am 19. 6. 1960 unabhängig (deren Verfassung: s. Hauptband S. 174). Nachdem Senegal am 20. 8. 1960 ausgetreten war, nahm der Gliederstaat Sudan am 22. 9. 1960 den Namen Mali an.

23. 1. 1959 Verfassung der sudanesischen Republik (in der Communauté unter Mali)

22. 9.1960 Gesetz Nr. 35 der Republik Sudan: Proklamation der Republik Mali

22. 9. 1960 Gesetz Nr. 1 der Republik Mali; Verfassung der Republik Mali Anderungen:

1) 20.1.1961, Gesetz Nr. 25, hebt Vizepräsident in Art. 8, 9, 12, 13, 23, 47 auf (J. O. v. 15. 3., S. 245 : Dekret Nr. 19 v. 22. 2.)

2) 20. 1. 1961, Gesetz Nr. 26, ändert Art. 1 V (J. O. v. 1. 3., S. 203: Dekret Nr. 11 v. 18. 2.) 
3) 13. 3. 1965, Verfassungsgesetz Nr. 1, ändert Art. 6, 43, ersetzt überall "Cour d'Etat" durch "Cour Suprême" (J. O. v. 15. 4., S. 167)

AMTL. TEXT: J. O. v. 29. 9. 1960, S. 111

ENGL. UBERS.: YHR 1960, S. 234 (Auszug); Peaslee ${ }^{3}$, Africa, S. 535 (Fass. 1961) CAS, S. 1863 (Auszug)

FRANZ. TEXT: Lavroff, S. 165; Muracciole, S. 101; RJP 1961, S. 325; NE Nr. 2739 v. 13. 1. 1961, S. 59; 2995 v. 30. 5. 1963, S. 22

ITAL. ÜBERS.: Foderaro Bd. 2, S. 295

RUSS. UBBERS.: KGA Bd. 1, S. 417

28. 11.1968

7. 7.1969

Ordonnance Nr. 1: Vorläufige Verfassung (Militärdiktatur) in Kraft: 19. 11. 1968

Änderungen: 28. 8. 1969, Ordonnance Nr. 47, ändert Art. 11 (J. O.v. 1.9.1969)

AMTL. TEXT: J. O. Sonder-Nr. 288 v. 28. 11. 1968, S. I

ENGL. ỦBERS.: CAS, S. 1858; CCW, Mali (1974)

FRANZ. TEXT: EPC (Jouve) 1974

Ordonnance Nr. 37: Schaffung eines „Council national consultatif"

AMTL. TEXT: J. O. v. 15.7. 1969

2. 6.1974 Verfassung der Republik

Veröffentlichung des Entwurfs: 25. 4. 1974; VA: 2. 6. 1974;

Proklamierung der Annahme: 18. 6. 1974

AMTL. TEXT: J. O. v. 25. 7. 1974, S. 1 (Dekret Nr. 3 v. 1. 7.74 betr. Verkündung)

FRANZ. TEXT: Verfassungstexte, Beilage zu VRÜ Nr. 2/1975

\section{MAROKKO}

Unabhängig von Frankreich durch Vertrag v. 2. 3. 1956.

2. 6. 1961 Dahir Nr. 167: Grundgesetz des Königreichs Marokko (Erste Verfassung) s. Hauptband S. 178

18.11.1962 (Zweite) Verfassung des Königreichs Marokko

Volksabstimmung: 7. 12. 1962

in $\mathrm{Kraft}:$ 7. 12. 1962-7.6.1965

AMTL. TEXT: B. O. v. 19. 12. 1962, S. 1773 (Dahir v. 14. 12. betr. Veröff.)

TEXTE: Hauptband S. 178

ferner:

ENGL. UBERS.: CAS, S. 1109; Peaslee ${ }^{3}$, Africa, S. 562; CP inf. 1963, S. 5

FRANZ. TEXT: JOOR 1972, S. 507; RJP 1963, S. 322; Inf. CP 1963, S. 5

ITAL. Übers.: Foderaro Bd. 2, S. 307; RI 1963, S. 335

RUSS. UBERS.: KGA Bd. 1, S. 479

SPAN. UBERS.: BLE 1963, S. 5 

Nr. 177 v. 31. 7.)

ENGL. UBBERS.: CCW, Morocco, 1971; ACR, 1970/1, C. 131 FRANZ. TEXT: Orient 1970, S. 172; Maghreb 1970, Nr. 41, S. 29; RJP 1971, S. 131; JÖR 1972, S. 515

10. 3.1972 SPAN. ÜBERS.: Inf. jur. Nr. 306 v. 1970, S. 63

(Vierte) Verfassung des Königreiches Marokko

Volksabstimmung: 1. 3. 1972

AMTL. TEXT: B. O. Nr. 3094 bis v. 18. 2. 1972, S. 233 (Referendumstext: Dahir Nr. 41 v. 11. 2.); B. O. Nr. 3098 v. 15. 3. 1972, S. 456 (Dahir Nr. 61 v. 10. 3.)

ENGL. UBBERS.: Peaslee ${ }^{4}$, Africa, S. 592

FRANZ. TEXT: JOOR 1972, S. 522

ITAL. UBERS.: O. M. 1972, S. 125 (Auszug)

\section{SONDERGEBIET: IFNI}

Das Territorium Ifni (s. Hauptband, S. 344-345) wurde durch Vertrag mit Spanien v. 4. 1. 1969 (Aranzadi 1969, Nr. 1053; B. O. Nr. 2952 v. 28. 5. 1969, S. 571: Dahir Nr. 61 v. 13. 5. 1969) an Marokko abgetreten, was am 13. 5. 1969 in Kraft trat.

19. 1. 1970 Dahir $1-69-326$ betr. Ausdehnung marokk. Gesetze auf Ifni AMTL. TEXT: B. O. Nr. 2986 v. 21. 1. 1970, S. 77

\section{MAURETANIEN}

Unabhängig von Frankreich am 28. 11. 1960.

22. 3. 1959 Verfassung der Islam. Republik Mauretanien

(bis 28.11. 1960 in der Communauté)

Änderungen:

1) 4. 6. 1960 , Verf. Gesetz Nr. 525 betr. Rechte in der Communauté (J. O. v. 15. 6. 1960, S. 315)

2) 26. 11. 1960, Gesetz Nr. 193 betr. Verfassungsänderung J. O. Nr. 46 v. 7. 12. 1960, S. 588)

AMTL. TEXT: J. O. Mauretanien v. 13. 5. 1959, S. 9; Weitere

Texte s. Hauptband S. 184 
20. 5. 1961

Verfassung der Islam. Republik Mauretanien, Gesetz Nr. 15

Änderungen:

1) 24.4. 1964, Verf. Gesetz Nr. 62, ändert Art. 55

(J. O. v. 20. 5., S. 115)

2) 12. 2. 1965, Gesetz Nr. 39, ändert Art. 9

(J. O. v. 3. 3., S. 61; YHR 1965, S. 207)

3) 12. 7. 1966, Gesetz Nr. 135, ändert Art. 13

(J. O. v. 3. 8., S. 214)

4) 4. 3. 1968, Gesetz Nr. 65, ändert Art. 3, 47, 53

(J. O. v. 27. 3., S. 106; YHR 1968, S. 665)

5) 21. 1. 1969, Gesetz Nr. 52, ändert Art. 53

(J. O. v. 29. 1. 69, S. 56)

6) 4. 6. 1970, Gesetz Nr. 182, ändert Art. 24, 27

(J. O. v. 27.6., S. 157)

AMTL. TEXT: J. O. Nr. 59 v. 3. 6. 1961, S. 171

ENGL. ÜBERS.: Peaslee ${ }^{3}$, Africa S. 549; CAS S. 979; CCW, Mauritania, 1973; Peaslee 4 , Africa, S. 503 (Fass. 1965)

FRANZ. TEXT: Lavroff S. 178; Muracciole S. 110; RJP 1967, S. 439; NE Nr. 2995 v. 30. 5. 1963, S. 28

ITAL. ÜBERS.: Foderaro Bd. 2, S. 321 (Fass. 1966)

RUSS. UBBERS.: KGA Bd. 1, S. 396

\section{MAURITIUS}

Unabhängig von Großbritannien am 12. 3. 1968.

Vorschriften bis 1960, Hauptband S. 311.

26. 2.1964 Mauritius (Constitution) Order in Council

Ainderungen:

1) 8. 11.1965 (S. I. 1920)

AMTL. TEXT: S. I. Bd. 1, S. 1163

21.12.1966 Mauritius (Constitution) Order in Council

Anderungen:

1) 12. 4. 1967 (S. I. 1967, Bd. 1, S. 2132)

2) 28. 7.1967 (S. I. 1967, Bd. 2, S. 3807)

3) 28. 11.1967 (S. I. 1967, Bd. 2, S. 5455)

AMTL. TEXT: S. I. 1966, Bd. 3, S. 5190

4. 3.1968 Mauritius Independence Order in Council

The Constitution of Mauritius (Schedule)

in Kraft: 12. 3. 1968

Änderungen:

1) 20.11. 1969 (A 39/69) ändert S. 57, 59, 66, 112

2) 13. 11.1973 (A 40/73) ändert S. 35, Schedule 1

AMTL. TEXT: S. I. Bd. 1, S. 1871; G. N. (Suppl. Gaz. v. 6. 3. 1968)

ENGL. TEXT: YHR 68, 270; CCW, 1971; Peaslee4, Africa, S. 516

FRANZ. ÜBERS.: EPC (Favoreau) 


\section{MOZAMBIQUE}

Úber die portugies. Úberseeprovinz bis 1955 s. Hauptband S. 335. Unabhängig seit 25. 6. 1975

22. 11.1963

22. 12.1972

15. 6.1974

24. 7.1974

7. 9.1974

9. 9.1974
Dekrret Nr. 45375: (2.) Statut für Mozambique

AMTL. TEXT: DdG Nr. 274, S. 1793

Dekret Nr. 545: (3.) Statut für Mozambique

AMTL. TEXT: DdG Nr. 296, Suppl. v. 22. 12. 1972, S. 2030 (31)

Dekret Nr. 255 betr. Staatssekretäre für Mozambique

AMTL. TEXT: DdG Nr. 138, S. 713

Gesetz Nr. 6 betr. Regierungsjunta für Angola und Mozambique AMTL. TEXT: DdG Nr. 171, Suppl., S. 846 (1)

Abkommen Portugals mit der FRELIMO über die schrittweise Unabhängigkeit

AMTL. TEXT: DdG Nr. 210 v. 9. 9. 1974, 2. Suppl., S. 1032 (3)

DT. ÜBERS.: EA 1974, D. 429

ENGL. TEXT: ILM 1974, S. 1467

PORT. TEXT: Diario de Noticias v. 9. 9. 1974

Gesetz Nr. 8 betr. Übergangsregime

AMTL. TEXT: DdG Nr. 210, 2. Suppl., S. 1032 (5)

\section{NAMIBIA/SÜDWESTAFRIKA}

Die deutsche Kolonie Südwestafrikas wurde 1920 südafrikanisches Völkerbundsmandat. Seit der Gründung der Vereinten Nationen ist die Rechtslage umstritten. Während Südafrika das Gebiet weitgehend als integralen Bestandteil behandelt, hat der Int. Gerichtshof entschieden, daß die Mandatspflichten nunmehr gegenüber der UN bestehen. Die UN-Vollversammlung hat Südafrika am 27. 10. 1966 das Mandat entzogen, am 19. 5. 1967 eine UN-Verwaltung eingesetzt, und das Gebiet am 12. 6. 1968 in Namibia umbenannt. Der UN-Sicherheitsrat (SC Res. 264 [1970]) und der Int. Gerichtshof (ICJ. Adv. Opinion [1971]) haben diese Akte bestätigt.

A. Südaf rikanische Vorschriften

s. Hauptband, S. 230

27. 7.1925 South West Africa Constitution Act dazu Änderungen:

6) 8.2. 1966 (A. 1/66)

29. 3.1968 South West Africa Constitution Act Änderungen:

1) 27. 2. 1970 (A. 13/70) ändert S. 14

AMTL. TEXT: A. 39/68; Statutes Class. Constitutional Law, S. 785

6. 6. 1968 Development of Self. Government for Native Nations in South West Africa Act

Änderungen: 30. 3. 1973 (A. 20/73)

AMTL. TEXT: A. 54/68; Statutes Class. Constitutional Law, S. 831 

B. UN-Dokumente
27. 10. 1966
Resolution der Generalversammlung
Entzieht Südafrika das Mandat
AMTL. TEXT: 2145 (XXI) GA Res.
19. 5.1967
Resolution der Generalversammlung
Setzt „United Nations Council for South West Africa“ zur interimistischen Verwaltung des Territoriums ein
AMTL. TEXT: 2248 (S- V) GA Res.
12. 6.1968
Resolution der Generalversammlung
Umbenennung des Gebiets in „Namibia“
AMTL. TEXT: 2372 (XXII) GA Res.

\section{NIGER}

Unabhängig von Frankreich am 3. 8. 1960.

12. 3.1959 Verfassung der Republik Niger (in der Communauté)

s. Hauptband S. 192

8.11.1960 Verfassung der Republik Niger

Änderungen:

1) 12.7. 1961, Gesetz Nr. 25, ändert Art. 31 u. 51 (J.O.v.31.8., S.67)

2) 14. 3. 1964, Gesetz Nr. 1, ändert Art. 64 (J. O. 21. 3., S. 1)

3) 14. 8. 1964, Gesetz Nr. 28, ändert Art. 31 u. 51 (J. O. v. 15. 8., S. 2)

4) 7. 9. 1965, Gesetz Nr. 34, ändert Art. 10 u. 29 (J. O. v. 15. 9., S. 2; YHR 1965, S. 219)

AMTL. TEXT: J. O. v. 8. 11. 1960

ENGL. UBERS.: Peaslee ${ }^{3}$, Africa S. 578 (Fass. 1960); CAS S. 1130 (Fass. 1961); CP Inf. 1967, S. 71 (Fass. 1965); CCW, Niger, 1973; Peaslee ${ }^{4}$, Africa, S. 609

FRANZ. TEXT: Lavroff, S. 102 (Änd. Nr. 1: Suppl. S. 43); Muracciole, S. 116; RJP 1961, S. 446; NE Nr. 2995 v. 30. 5. 1963, S. 33; Inf. CP 1967, S. 102 (Fass. 1965)

ITAL. UBERS.: Foderaro Bd. 2, S. 401 (Fass. 1960)

RUSS. UBBERS.: KGA Bd. 1, S. 504 (Fass. 1961)

22. 4. 1974 Ordonnance Nr. 1 betr. Außerkraftsetzung der Verfassung und Einsetzung eines Conseil Militaire Suprême 


\section{NIGERIA}

Unabhängig von Großbritannien am 1. 10. 1960 als Bundesstaat mit drei Regionen und Bundesterritorium Lagos. Am 1. 10. 1963 Republik. Nach Militärputsch 1966 Einheitsstaat. Ein zweiter Putsch im selben Jahr stellte bundesstaatliche Ordnung wieder her. 1967 wurden die alten Regionen durch ein 12-Staaten-System ersetzt. Die ehemalige Ostregion erklärte sich am 30. 5. 1967 unter dem Namen Biafra für unabhängig. Nach einem 30monatigen Bürgerkrieg wurde sie wieder in den Bundesstaat eingegliedert.

12. 9. 1960 Constitution of the Federation of Nigeria

Hauptband, S. 194, dazu Änderungen:

1. 10. 1963 Constitution of the Federal Republic of Nigeria AMTL. TEXT: A. 20/63

ENGL. TEXT: Peaslee $^{3}$ I, S. 592

FRANZ. UBERS.: Lavroff II, S. 149

17. 1.1966 Constitution (Suspension and Modification) Decree

Änderungen:

1) 17.12 .1966 (D. $14 / 66)$

2) 28. 3. 1966 (D. $17 / 66)$

3) 4. 4.1966 (D. 20/66)

4) 24. 5. 1966 (D. 34/66) Einheitsstaat

5) 2. 6.1966 (D. $36 / 66)$

6) 2. 7.1966 (D. 50/66)

7) 8. 8.1966 (D. 55/66)

8) 1. 9.1966 (D. 59/66) wieder Bundesstaat

9) 10.12 .1966 (D. 69/66)

AMTL. TEXT: D. 1/66 (Gaz. Suppl. No. 20 v. 4. 3. 1966) ENGL. TEXT: von Nr. 4 und 8 CCW (1972)

17. 3.1967 Constitution (Suspension and Modification) Decree

(hebt D. 1/66 und Änderungen 1-9 auf und veröffentlicht sie in konsolidierter Form)

AMTL. TEXT: D. $8 / 67$

ENGL. TEXT: CCW (1972)

26. 5.1967 Constitution (Repeal and Restoration) Decree

Hebt D. 8/67 auf und stellt die Verfassungslage auf der Grundlage von D. 1/66 (einschließlich der Änderung 1-9) wieder her, die gleichzeitig geändert werden.

Anderungen:

1) 30.5 .1967 (D. 16/67)

2) 8.6 .1967 (D. 20/67)

3) 12.7 .1967 (D. 27/67)

4) 21.8 .1968 (D. 44/68)

5) 22. 9. 1969 (D. 42/69)

AMTL. TEXT: D. 13/67

ENGL. TEXT: CCW (1972) 
a) North-Western

b) North-Central

c) Kano

d) North-Eastern

e) Benue-Platea

f) Central-West

g) Lagos

h) Western

i) Mid-Western

j) Central-Eastern

k) South-Eastern

l) Rivers

Das Decree enthält auch die für sämtliche Staaten geltende Verfassungsbestimmungen.

Änderungen:

1) 29.6 .1967 (D. 25/67)

AMTL. TEXT: D. $14 / 67$

ENGL. TEXT: CCW (1972)

9. 5.1970 Federal Military Government (Supremacy and Enforcement of Powers) Decree

AMTL. TEXT: D. 28/70

ENGL. TEXT: CCW (1972)

\section{SONDERGEBIET: BIAFRA}

30. 5. 1967 Unabhängigkeitserklärung

ENGL. TEXT: Keesings 22 088; ILM 1967, S. 665

\section{OBERVOLTA}

Unabhängig von Frankreich am 5. 8. 1960.

2. 3.1959 (Erste) Verfassung der Republik (in der Comunauté)

s. Hauptband S. 200

9.11. 1960 (Zweite) Verfassung der Republik, Gesetz Nr. 86

VA: 27. 11. 1960

Änderungen:

1) 29. 1. 1963, Gesetz Nr. 463, ändert Art. 64

(J. O. v. 16. 2., S. 118)

2) 28. 7. 1965, Gesetz Nr. 12, ändert Art. 11 u. 29

(J. O. v. 29. 7., S. 354)

AMTL. TEXT: J. O. Nr. 44 bis v. 12. 11. 1960, S. 1 (Dekret Nr. 437: Referendumstext); J. O. Nr. 47 bis v. 3. 12. 1960 (Dekret Nr. 475 v. 30. 11. = Verkündungstext) 
DT. UBERS.: Afrika Inf. dient v. 15. 12. 1960, Dok. 78

ENGL. UBERS.: YHR 1960, S. 372 (Auszug); Peaslee ${ }^{3}$, Africa S. 1012 (Fass. 1960)

FRANZ. TEXT: Lavroff, S. 90; Muracciole, S. 75; RJP 1961, S. 299; NE Nr. 2995 v. 30. 5. 1963, S. 7

ITAL. UBERS.: Foderaro Bd. 1, S. 11 (Fass. 1960)

RUSS. UBERS.: KGA Bd. 1, S. 51 (Fass. 1963)

5. 1.1966 Ordonnance Nr. 1: Außerkraftsetzung der Verfassung von 1960

AMTL. TEXT: J. O. Nr. 1 v. 6. 1. 1966, S. 1

FRANZ. TEXT: RJP 1966, S. 358

19. 5.1970

(Dritte) Verfassung der Republik

VA: 14. 6. 1970

Proklamation der Annahme durch Ordonnance v. 24. 6. 1970 (J. O. v. 25. 6., S. 378)

AMTL. TEXT: J. O. Nr. 21 v. 19. 5. 1970, S. 4 (Entwurftext: Dekret Nr. 93); J. O. Nr. 7 v. 18. 2. 1971, S. 129 (Verkündungstext: Dekret Nr. 125 v. 29. 6. 70)

ENGL. UBERS.: YHR 1970, S. 260 (Auszug); CCW, Upper Volta, 1971; Peaslee ${ }^{4}$, Africa, S. 1005

FRANZ. TEXT: RJP 1971, S. 165

\section{REUNION}

Franz. Uberseedepartement. Siehe Hauptband S. 282 f. Keine neuen Regelungen seit 1947.

\section{RHODESIEN}

Seit 1923 ist Süd-Rhodesien britische Kronkolonie mit weitgehender Selbstverwaltung. 1953-1963 Teil der Föderation von Rhodesien und Nyasaland. Am 11. 11. 1965 einseitige Unabhängigkeitserklärung der von der weißen Minderheit gestellten Regierung. Am 23. 3. 1970 Erklärung zur Republik. Die Unabhängigkeitserklärung wird von Großbritannien nicht anerkannt.

Vorschriften vor 1961, Hauptband S. 317.

6. 12. 1961 Southern Rhodesia (Constitution) Order in Council Anderungen:

1) 16. 5. 1964 (A. 13/64) ändert S. 14, 43, 61, 63, 67, 71, 74, 76, $77,80,81,94,96,108,116,117$, sch. 1,3

2) 23. 6. 1964 (S. I. 923) ändert, S. 1 (1)

3) 1. 10.1965 (A. 41/65) ändert, S. 10, 11, 13, 14, 23, 28, 33, 43, $45,48,77,113,117$, sch. 1 , hebt auf, S. 46

AMTL. TEXT: S. I. 2314; Statute Law of Southern Rhodesia, 1963, Bd. 1, S. 27

6. 6.1962 Instructions to the Governor

AMTL. TEXT: Statute Law of Southern Rhodesia, 1963, Bd. 1, S. 109 


\section{A. Britische Vorschriften}

16. 11.1965

\section{Southern Rhodesia Act}

(Nichtanerkennung der einseitigen Unabhängigkeitserklärung und weitere Verwaltung durch Großbritannien)

AMTL. TEXT: 1965 c. 76

16. 11.1965

\section{Southern Rhodesia (Constitution) Order in Council}

Regelt Verwaltung durch Großbritannien; wird jährlich verlängert (1966, S. I. 1407; 1967, S. I. 1674; 1968, S. I. 1697; 1969, S. I. 1504; 1970, S. I. 1702; 1971, S. I. 1847; 1972, S. I. 1717) AMTL. TEXT:

\section{B. Rhodesische Vorschriften}

11.11 .1965

\section{Constitution of Rhodesia}

Anderungen:

1) 16. 9. 1966 (A. 49/66) ändert S. 67, 70, 72, 76, 81, 101, 102, $104,108,110,134,144,145$, fügt ein 78 A u. 78 B

2) 15.11 .1968 (A. 43/68) ändert S. 48, 90

3) 31. 1. 1969 (A. 1/69) ändert S. 81

4) 19. 9. 1969 (A. 42/69) ändert S. 114

AMTL. TEXT: Procl. Nr. 53/1965; G. N. 737; Constitution (Ratification) Act (Schedule) 1966, Act 1/66

5. 12.1969 Constitution of Rhodesia

Republikanische Verfassung

Volksabstimmung 20. 6. 1969

in Kraft: 2. 3. 1970

AMTL. TEXT: A.54/69

ENGL. TEXT: CCW (1973); Peaslee ${ }^{4}$, Africa, S. 771

FRANZ. UBERS.: NE 4151

\section{RWANDA}

Unabhängig von Belgien (Treuhänder) a m 1. 7. 1962, nachdem sich das Königreich Ruanda schon am 29. 1. 1961 zur Republik erklärt hatte.

28. 1. 1961 Verfassung der Republik Rwanda

AMTL. TEXT: nicht veröffentlicht, da nicht formell in $\mathrm{Kraft}$ gesetzt

FRANZ. TEXT: Rev. jur. du droit écrit et coutumier du Rwanda et du Burundi Nr. 2 v. 1961

24. 11.1962

\section{Verfassung der Republik Rwanda}

Anderungen:

1) 12. 6. 1963, ändert Art. 108 (J. O. Nr. 14 v. 15. 7., S. 329)

2) 18. 5. 1973, ändert Art. 44, 48-49, 52-56, 64, 70, 73, 97

(J. O. Nr. 11 v. 1. 6., S. 133) 
AMTL. TEXT: J. O. Nr. 22 bis v. 1. 12. 1962, S. 439; J. O. Nr. 11 bis v. 10. 6. 1973 , S. 151 (Neuverkündung i. d. F. d. Änderungen v. 1963 u. 1973)

ENGL. ÜBERS.: Peaslee ${ }^{3}$, Africa, S. 675; CAS S. 1316; CCW, RWANDA, 1973; Peaslee ${ }^{4}$, Africa, S. 627

FRANZ. TEXT: CPE 1963, S. 646; NE Nr. 3175 v. 26. 3. 1965, S. 15

ITAL. ÜBERS.: Foderaro Bd. 2, S. 503

RUSS. UBBERS.: KGA Bd. 3, S. 177

5. 7.1973 Außerkraftsetzen der Verfassung durch Staatsstreich

40. SAHARA (SPANISCH-WESTAFRIKA)

Bis 1958 s. Hauptband S. 344. Uber Abtretung v. Ifni (1969) s. hier Marokko.

19. 4.1961 Gesetz Nr. 8: Organisation der Rechtsordnung

AMTL. TEXT: BOE Nr. 95 v. 21. 4. 1961

SPAN. TEXT: Aranzadi 1961, Nr. 577

29.11. 1962 Dekret Nr. 3249: Ordnung der Verwaltung der Provinz Sahara AMTL. TEXT: BOE Nr. 297 v. 12. 12. 1962

SPAN. TEXT: Aranzadi 1962, Nr. 2218

11. 5.1967 Dekret Nr. 1024: Ergänzung der Verwaltungsordnung

hängt ein neues Kapitel (Art. 164-178) an.

AMTL. TEXT: BOE Nr. 120 v. 20. 5. 1967

SPAN. TEXT: Aranzadi 1967, Nr. 948

\section{Saint HELENA}

Bis 1956 s. Hauptband S. 319

22. 11.1966

St. Helena Constitution Order in Council

Constitution of St. Helena (Schedule 1)

Änderungen:

1) 28. 7. 1967 (S. I. 1138), ändert S. 8 des Order und S. 49 der Verfassung

22. 11.1966

AMTL. TEXT: S. I. 1458

Royal Instructions

AMTL. TEXT: S. I. Bd. 3, S. 5186

\section{SAO TOME E PRINCIPE}

Die portugies. Úberseeprovinz s. bis 1955 Hauptband S. 340.

Unabhängig 12. 7. 1975.

22. 11.1963

Dekret Nr. 45373 : (2.) Statut für S. Tomé e Principe

AMTL. TEXT: DdG Nr. 274, S. 1778

22. 12. 1972 Dekret Nr. 543: (3.) Statut für S. Tomé e Principe

AMTL. TEXT: DdG Nr. 296 v. 22. 12. 1972

26. 11. 1974 Abkommens Portugals mit der Unabhängigkeitsbewegung für

S. Tomé e Principe

ENGL. TEXT: ILM 1975, S. 39 (UN Doc. A 9953 v. 17. 12. 74) 


\section{SENEGAL}

Die Föderation Mali, zu der Senegal gehörte, wurde am 19. 6. 1960 von Frankreich unabhängig. Am 20. 8. 1960 trat Senegal aus der Föderation aus und wurde ein eigener Staat.

24. 1. 1959

(Erste) Verfassung der Republik Senegal in der Mali-Föderation (in der Communauté)

26. 8. 1960 (Zweite) Verfassung der Republik, Gesetz Nr. 45

Anderungen:

1) 12. 11. 1961, Gesetz Nr. 63, ändert Art. 35, 39, 53, 54

(J. O. v. 14. 11.)

2) 18. 12. 1962, Gesetz Nr. 62, fügt Art. 66 bis ein (J. O. 19. 12., S. 1939)

3) 4. 1. 1963, Gesetz Nr. 2, ändert Art. 64-65 (J. O. v. 7. 1., S. 21

AMTL. TEXT: J. O. (Senegal) Nr. 3396 v. 31. 8. 1960, S. 881 (Dekret Nr. 286); J. O. (Communauté) v. 15. 10. 1960, S. 143 ENGL. ÜBERS.: YHR 1960, S. 298 (Auszug)

FRANZ. TEXT: Lavroff, S. 190 (Änderung Nr. 1-3: Suppl., S. 44-47); Muracciole, S. 124; RJP 1961, S. 453 (Änderung Nr. 1: S. 619); NE Nr. 2754 v. 22. 1. 1961, S. 42

7. 3. 1963 (Dritte) Verfassung der Republik, Gesetz Nr. 22

VA: 3. 3. 1963

Änderungen:

1) 20. 6. 1967, Gesetz Nr. 32, ändert Art. 22, 24, 31, 45, 47, 49, 67, 88, 89; fügt ein Art. 53 bis, 75 bis (J. O. v. 10. 7., S. 1033; YHR 1967, S. 288; RJP 1968, S. 106; DLAA 1968, Nr. 1, S. 6).

2) 14. 3. 1968, Gesetz Nr. 4, ändert Art. 9 (J. O. v. 30. 3., S. 368)

3) 26. 2. 1970, Gesetz Nr. 15, ändert Präambel, Art. 5, 21, 22, 26, $33-39,43-46,49,52,56,57,65-66,70-72,74,75,75-80$, 88-89 (J. O. v. 28. 2., S. 230; YHR 1970, S. 200)

AMTL. TEXT: J. O. v. 11. 3. 1963, S. 357

ENGL. UBBERS.: Peaslee $^{3}$, Africa, S. 697; CP Inf. 1963, S. 81 (Änderung Nr. 3: 1971, S. 34); Peaslee ${ }^{4}$, Africa, S. 649

(Fass. 1970)

FRANZ. TEXT: NE Nr. 2995 v. 30. 5. 1963, S. 40; Inf. CP 1963

S. 80 (Änderung Nr. 1: 1969, S. 157; Nr. 3: 1971, S. 56); RJP 1970, S. 292 (Fass. 1970); RDP 1963, S. 229; JO'R 1971, (Bd. 20), S. 628 (Fass. 1970)

ITAL. ÜBERS.: Foderaro Bd. 3, S. 1

RUSS. UBERS.: KGA Bd. 3, S. 218 


\section{SEYCHELLEN}

Unabhängig von Großbritannien für Juni 1976 geplant (ARB 1975, 3572 B).

Vorschriften bis 1960 Hauptband, S. 320.

8. 4. 1960 Seychelles (Legislative Council) Order in Council

Anderungen:

1) 29. 7. 1963 (S. I. Bd. 2, S. 2775) ändert S. 2, 5, 10, 11, 12, 18,29

2) 10. 10. 1967 (S. I. Bd. 3, S. 5416) ändert S. 29 (2)

AMTL. TEXT: S. I. 1960, S. 4201

2. 5.1960 Additional Instructions to the Governor

AMTL. TEXT: S. I. 1960 Bd. 3, S. 4212

13.11.1967 Seychelles Order in Council

inKraft: 27. 11. 1967

Änderungen:

1) 22. 10.1969 (S. I. 1969 Bd. 3, S. 5859) ändert S. 67

AMTL. TEXT: S. I. 1967 Bd. 3, S. 5423

21.11.1967 Seychelles Royal Instructions

AMTL. TEXT: S. I. 1967 Bd. 3, S. 5452

30. 9.1970 Seychelles Order in Council

AMTL. TEXT: S. I. 1970 Bd. 3, S. 6728

\section{SIERRA LEONE}

Am 27. 4. 1961 unabhängig von Großbritannien.

Am 19. 4. 1971 Republik.

14. 4. 1961 Sierra Leone (Constitution) Order in Council

Constitution of Sierra Leone (Schedule)

Anderungen:

1) 25. 8. 1961 (A. 38/61) ändert S. 7

2) 17. 3. 1962 (A. 11/62) ändert S. 2, 3, 10, 31

3) 17. 3. 1962 (A. 12/62) ändert S. 1, 4, 7

4) 3. 10. 1962 (A. 39/62) ändert S. 23

5) 17. 5. 1965 (A. 4/65) ändert S. 7

6) 15.6 .1965 (A. 20/65) ändert S. 51

8) 31. 12. 1965 (A. 52/65) konsolidiert Änd. Gesetze und ändert S. 53;

(Suspendiert v. 25. 3. $1967-18.4$. 1968) s. unten

9) 14. 1. 1969 (A 2/69) ändert S. 59

10) 16. 4. 1971 (A. 4/71) ändert S. 59

AMTL. TEXT: S. I. 741 ; P. N. 78/61

ENGL. TEXT: Peaslee $^{3}$ I. S.

FRANZ. UBERS.: NE 3574; Lavroff, S. 304 
25. 3.1967

Administration of Sierra Leone

(National Reformation Council) Proclamation

Militärregierung

Ainderungen:

1) 26. 4. 1967 (NRC Law 7/67)

AMTL. TEXT: P. N. 28/67

ENGL. TEXT: ALD 3 (1967), S. 424

18. 4. 1968 Administration of Sierra Leone (National Interim Council)

Proclamation

Übergangsregierung für Rückkehr zur Zivilregierung

Änderungen:

1) 23. 4. 1968 (D. 2/68)

AMTL. TEXT: P. N. 21/68

ENGL. TEXT: ALD 3 (1967), S. 427

26. 4.1968 Administration of Sierra Leone

(Return to Civilian Rule) Proclamation)

AMTL. TEXT: P. N. 24/68

ENGL. TEXT: ALD 3 (1967), S. 427

19. 4.1971 Constitution of Sierra Leone

Anderungen:

1) 21. 4. 1971 (A. $7 / 71$ ) ändert S. $16,17,18,27,49,58,62,64$

2) 30. 5. 1972 (A. 13/72) ändert S. 28

AMTL. TEXT: A. 6/71

ENGL. TEXT: CCW (1973); Peasle4, Africa, S. 670 (Fass. v. 21. 4. 1971)

24. 5. 1971 Constitution (Consequential Provisions) Act

in Kraft: 19. 4. 1971

AMTL. TEXT: A 9/71

ENGL. TEXT: CCW (1973)

\section{SOMALIA}

Das italienische Treuhandgebiet Somalia und das britische Protektorat Somaliland vereinigten sich bei Erlangung der Unabhängigkeit 1960 zur Republik Somalia.

1. 7.1960 Verfassung von Somalia

Hauptband, S. 222; dazu

ENGL. TEXT: CCW (1971); YHR 1960, S. 301; CP Inf. 1964, S. 86

ITAL. TEXT: Angeloni 1964, S. 252

FRANZ. ÜBERS.: NE 3132; Inf. CP 1964, S. 92

DEUTSCHE UBBERS.: Süßner 1970, S. 133

21. 10. 1969 First Charter of the Revolution

AMTL. TEXT: Somalia Inf. Service 
Überträgt dem Supreme Revolutionary Council alle Staatsgewalt und suspendiert die Verfassung soweit sie der revolutionären Ordnung widerspricht.

AMTL. TEXT (Ital. u. Engl.): Official Bulletin v. 29. 10. 1969, Suppl. 1

24. 2. 1970 Decree (Decreto) No. 38

(Suspendiert die Verfassung)

AMTL. TEXT (Ital. u. Engl.): Official Bulletin (Bolletino Ufficiale) v. 1. 3. 1970, S. 130

\section{SUDAN}

Der Sudan war von 1899 bis 1955 britisch-ägyptisches Kondominimum und wurde am 1.1. 1956 unabhängig.

Vorschriften bis 1958 Hauptband, S. 233.

\section{11. 1958 Constitutional Order 1-3}

Suspendieren Verfassung von 1956 nach Militärcoup und regeln vorläufige Verfassung

20. 12.1962

ENGL. TEXT: YHR 1958, S. 209

vorläufige Verfassung

Transitional Constitution (Amended)

Stellt Verfassung von 1956 (Hauptband, S. 231) geändert wieder her

25. 5.1969

ENGL. TEXT: YHR 1965, S. 287

\section{Republican Order 1}

Republican Order 2

Republican Order 3

(Suspendieren Verfassung und regeln Staatsverwaltung)

AMTL. TEXT: Special Leg. Supplement to the Democratic; Republic of the Sudan Gazette No. 1076

13. 8.1971

Republican Order 5 vorläufige Verfassung

ENGL. UBERS.: Peaslee 4 , Africa, S. 835

14. 4.1973 Permanent Constitution of the Democratic Republic of Sudan

AMTL. TEXT: Democratic Republic of Sudan v. 8.5.73

ENGL. TEXT: CCW (1973)

\section{SƯDAFRIKA}

Britisches Dominion Südafrikanische Union seit 31. 5. 1910. Seit 31. 5. 1961 Republik Südafrika. Seit 1963 werden im Rahmen der südafrikanischen Apartheidpolitik „Homelands“ für die afrikanische Bevölkerung als „Self-governing territories" im Rahmen der Republik geschaffen.

Vorschriften vor 1962, Hauptband S. 224. 


\section{A. Gesamtstaat}

24. 4. 1961 Republic of South Africa Constitution

Anderungen:

1) 13.3 .1962 (A. 28/62) ändert S. 77

2) 9. 6. 1962 (A. 65/62) ändert S. 21

3) 5. 3. 1963 (A. 9/63) ändert S. 108

4) 23. 3. 1963 (A. 22/63) ändert S. 76

5) 15.5 .1963 (A. 47/63) ändert S. 84

6) 21. 3.1963 (A. 64/63) ändert S. 74, 76

7) 18.6 .1965 (A. $83 / 65)$ ändert S. 40, 42, 43

8) 21. 6. 1965 (A. 102/65) ändert S. 15

9) 5. 10.1966 (A. 37/66) ändert S. 62

10) 24. 10. 1966 (A. 58/66) ändert S. 104 A

11) 10. 2. 1967 (A. 9/67) ändert S. 8 sowie $12,20,52 \mathrm{der}$ Afrikaans Fassung

12) 1971 (A. 93/71) ändert S. 15

13) 1973 (A. 79/73) ändert S. 108

AMTL. TEXT (Engl. und Afrikaans): A. 32/61; Statutes Class., Constitutional Law, S. 411

ENGL. TEXT: CCW (1971); YHR 61, S. 311; JÖR Bd. 15, S. 630; CP Inf. 1962, S. 224; St. P. 166, S. 363; Corpus I, S. 85; Peaslee $^{3}$ I, S. 808; Peaslee ${ }^{4}$, Africa, S. 729

AFRIKAANS TEXT: CCW (1971); Corpus I, S. 83

FRANZ. UBERS.: NE 2928; Inf. CP 1962, S. 218; Lavroff II, S. 68

ITAL. ỦBERS.: Foderaro III, S. 103

\section{B. Homelands}

(Während die Transkei durch Gesetz gegründet wurde, werden weitere Homelands aufgrund des Bantu Homelands Constitutions Act durch Proklamation der Staatspräsidenten gegründet.)

\section{Transkei}

30. 5.1963 Transkei Constitution Act

Anderungen:

1) 19. 6. 1967 (A. 101/67) ändert S. 23, 25, 26, 29, 45, 49, 73

2) 28.3 .1968 (A. 36/68) ändert S. 4, 8, 50, Sch. 1

3) 5. 5. 1971 (A. 31/71) ändert S. 12, 15, 17, 18, 19, 23, 25

4) $1972($ A. 23/72)

5) $1973($ A. $7 / 73)$

AMTL. TEXT

(Englisch und Afrikaans): A. 48/63 
2. Gemeinsame Vorschriften für die übrigen Homelands

31. 3.1971 Bantu Homelands Constitutions Act

Änderung:

1) 1972 (A. 23/72) ändert Sch. 1

2) 1973 (A. 7/73)

AMTL. TEXT

3. Bophuthatswana

(Englisch und Afrikaans): A. 21/71

26. 5. 1972 Bophuthatswana Constitution Proclamation

in Kraft: 1. 6. 1972

4. Ciskei

AMTL. TEXT: P. R. 131 (Reg. Gaz. 1630)

28. 7.1972

Ciskei Constitution Proclamation

in Kraft: 1. 8. 1972

AMTL. TEXT: P. R. 187 (Reg. Gaz. 1650)

5. Lebowa

22. 9.1972

Lebowa Constitution Proclamation

AMTL. TEXT: P. R. 224 (Reg. Gaz. 1762)

6. Venda

26. 1.1973

Venda Constitution Proclamation

7. Gazankulu

AMTL. TEXT: P. R. 11/2 (Reg. Gaz. 1733)

26. 1.1973

Gazankulu Constitution Proclamation

AMTL. TEXT: P. R. 14/15 (Reg. Gaz. 1735)

\section{SWAZILAND}

Das britische Protektorat Swaziland wurde am 1. 3. 1967 Protected State und am 6. 9. 1968 unabhängig. Am 12. 4. 1973 hob König Sobhuza II die Verfassung auf und übernahm die volle Regierungsgewalt.

20.12. 1963 Swaziland Order in Council

Änderungen:

1) 27. 7. 1964 S. I. 1192

2) 24.6 .1965 S. I. 1305

3) 20.9. 1966 S. I. 1178

AMTL. TEXT: S. I. 2094

22. 2. 1967 Swaziland (Constitution) Order in Council

Änderungen:

1) 28. 6. 1967 (S. I. 975) ändert S. 59, 115

2) 8. 5.1968 (S. I. 727) fügt ein S. 10 A, $10 \mathrm{~B}$

AMTL. TEXT: S. I. 241 
26. 8.1968

12. 4.1973

4. 5.1973
Swaziland Independence Order. Constitution of Swaziland

(Schedule)

in Kraft: 6. 9. 1968

Änderungen:

1) 17.7 .1970 (A. 36/70) ändert S. 99

AMTL. TEXT: S. I. 1377; A. 50/1968 c

ENGL. TEXT: CCW (1971); Peaslee ${ }^{4}$, Africa, S. 847

Proklamation des Königs

Aufhebung der Verfassung

Ubernahme aller Staatsgewalt durch den König

AMTL. TEXT: Swaziland Government Gazette v. 17. 4. 1973, S. 148

ENGL. TEXT: African Law Digest Bd. 9/1, S. XI

Legislative Procedure Order in Council

AMTL. TEXT: O. 1/73

\section{TANZANIA}

Die Vereinigte Republik Tanzania entstand am 27. 4. 1964 aus einer Vereinigung der bis dahin unabhängigen Staaten Tanganjika und Zanzibar. Zanzibar behielt eine weitgehende Autonomie, während für das ehemalige Tanganjika (MainlandTanzania) keine eigenen Verfassungsorgane und -vorschriften mehr gelten.

A. Gesamtstaat

25. 4.1964 Union of Tanganjika and Zanzibar Act

(Gesetz von Tanganjika. Der Unionsvertrag v. 22. 4. 64 ist Anlage, ratifiziert Unionsvertrag $\mathrm{m}$. W. v. 26. 4. 64)

AMTL. TEXT: A. 22/64 (Tanganjika)

ENGL. TEXT: Peaslee ${ }^{3}$ I, S. 1106; ILM 1964, S. 401

DEUTSCHE UBERS.: Afrika heute 1964, Dok. Nr. 136

25. 4.1964 Union of Zanzibar and Tanganjika Law

(Gesetz von Zanzibar, der Unionsvertrag v. 22. 4.64 ist Anhang)

AMTL. TEXT: G. N. 243

ENGL. TEXT: ILM 1964, S. 401

DEUTSCHE UBERS.: Afrika heute 1964, Dok. 136

26. 4. 1964 Interim Constitution Decree. Interim Constitution of the United Republik of Tanganjika and Zanzibar

(Ergänzt und ändert die Verfassung von Tanganjika, 1962 (CA 1/62) und nennt sie "Interim Constitution of the United Republic of Tanganjika and Zanzibar")

Anderungen:

1) 1964 (G. N. 266/64)

2) 19.6. 1964 (G. N. 360/64) ändert S. 46

3) 18.9 .1964 (G. N. A. 44/65)

4) 16.4 .1965 (G. N. 181/65) fügt ein $15,53 \mathrm{~A}$

5) 10.6 .1965 (A. 21/65) ändert S. 68

AMTL. TEXT: G. N. 246/64

ENGL. TEXT: ILM 1964, S. 410 
26. 4.1964 Transitional Provisions Decree

AMTL. TEXT: G. N. 245/64

ENGL. TEXT: ILM 1964, S. 408

29. 10. 1964 United Republic (Declaration of Name) Act

AMTL. TEXT: A. 61/1964

8. 7.1965 Interim Constitution of Tanzania

in Kraft: 11. 7. 1965

(Schedule 1 enthält das TANU-Statut)

Änderungen:

1) 7. 1.1966 (A. 4/66 ändert), S. 2

2) 9.8 .1967 (A. 35/67) ändert S. 85

3) 27.11 .1967 (A. 40/67) ändert S. 27, 31, 34 A, 34 B, 35, 37

4) 1968 (A. 36/68) ändert S. 68

5) 25. 7.1968 (A. 48/68) ändert S. 85

6) 10.10 .1968 (A. 56/68) ändert S. 24

7) 1971 (A. 28/71) ändert S. 58

8) 1971 (A. 29/71) ändert S. 21

9) 11. 5. 1972 (A. 10/72) ändert S. 24

10) 23.11. 1972 (A. 29/72) ändert S. 9

11) 23. 11.1972 (A. 32/72) ändert S. 56

AMTL. TEXT: A. 43/65; Revised Edition, Cap. 596, Suppl. 66/ 72; (Fassung vom 31.12. 1972)

ENGL. TEXT: CCW (1973) (Revised Edition); JO'R 1967, S. 610; CAS, S. 1517; Peaslee ${ }^{4}$, Africa, S. 926

FRANZ. ÜBERS.: EPC (Urfer) 1973

ITAL. UBERS.: Foderaro III, S. 189

8. 7. 1965 Interim Constitution (Consequential, Transitional, and

Temporary Provisions) Act

AMTL. TEXT: A. 45/65; Revised Edition c. 598, Suppl. 65/69

B. Tanganjika (Vorschriften bis zur Vereinigung)

Vorschriften bis 1961, Hauptband, S. 239.

23. 11. 1962 Constitution of Tanganjika

in Kraft: 9. 12. 1962

Änderungen:

1) 30.1. 1964 (A. 42/64) ändert S. 44

2) 17.9 .1964 (A. 44/64) ändert S. 47

AMTL. TEXT: Constituant Assembly Act 1/1962

ENGL. TEXT: Peaslee $^{3}$ I, S. 860

FRANZ. UBBERS.: Lavroff II, S. 361 
Vorschriften bis 1961, Hauptband S. 329.

C. Zanzibar

8. 12. 1924 Zanzibar Order in Council

dazu Änderungen:

10) 19. 12.1962 (S. I. 2784)

11) 30. 5. 1963 (S. I. 1042)

27.11.1963 Constitution of the State of Zanzibar

AMTL. TEXT: Gaz. Leg. Suppl. I No. 4320

3. 12. 1963 Zanzibar Act (Großbritannien)

AMTL. TEXT: $11 / 2$ Eliz. 2, C. 55

25. 2. 1964 Constitutional Government and the Rule of Law Decree

AMTL. TEXT: D. 5/64

12. 5. 1965 Afro-Shirazi

Party Decree

AMTL. TEXT: D. 11/65

1. 8.1972 Appointment of Chairman Decree

AMTL. TEXT: D. 8/72

\section{TOGO}

Unabhängig von Frankreich als Treuhänder am 27. 4. 1960.

14. 4. 1961 Verfassung der Republik Togo

außer Kraft 18. 1. 1963

AMTL. TEXT: J. O. Nr. 157 v. 17. 4. 1963, S. 293

FRANZ. TEXT: Lavroff, S. 207; Muracciole, S. 152; RJP 1961, S. 481 ; NE Nr. 2995 v. 30. 5. 1963, S. 57

11. 5. 1963 Verfassung der Republik Togo

VA: 5. 5. 1963

außer Kraft: 13. 1. 1967

Anderung: 15. 12. 1966, hebt auf Art. 21 und ändert daher alle Vorschriften über Vizepräsidenten insb. Art. 22, 24, 37, 40, 61 $65,71,83,86,87$ (J. O. v. 31. 12., Sonder-Nr. 342, S. 13) AMTL. TEXT: J. O. v. 12. 5. 1963, S. 305

ENGL. UBERS.: Peaslee ${ }^{3}$, Africa S. 890; CP Inf. 1964, S. 185; YHR 1963, S. 307 (Auszug)

FRANZ. TEXT: NE Nr. 3175 v. 26. 3. 1965, S. 25; Inf. CP 1964, S. 194; DLAA 1964, Nr. 1, S. 1

ITAL. UBERS: Foderaro Bd. 3, S. 249

RUSS. UBERS.: KGA Bd. 3, S. 418

SPAN. UBERS.: Inf. jur. Nr. 266/7 v. 1965, S. 1

14. 1.1967 Ordonnance Nr. 1: Aufhebung der Verfassung u. Bildung eines Rats der nat. Versöhnung

AMTL. TEXT: J. O. Nr. 345 v. 30. 1. 1967, S. 1

ENGL. UBERS.: CCW, Togo, 1974 
14. 4. 1967 Ordonnance Nr. 15: Präsident der Republik

AMTL. TEXT: J. O. v. 15. 4. 1967, S. 9

ENGL. ÜBERS.: a. a. O.

14. 4.1967 Ordonnance Nr. 16: Auflösung des Rats der nat. Versöhnung und Bildung einer Regierung

AMTL. TEXT: J. O. v. 15. 4. 1967, S. 9

ENGL. ÜBERS.: a. a. O.

8. 9. 1968 Entwurf einer Verfassung

ausgearbeitet vom Komitee auf Grund Ordonnance Nr. 22 v. 30. 5. 1967 (J. O. Sonder-Nr. v. 30. 6., S. 2), abgelehnt durch Referendum v. Jan. 1969

\section{TSCHAD}

Unabhängig von Frankreich am 11. 8. 1960.

31. 3.1959 (Erste) Verfassung der Republik Tschad (in der Communauté) s. Hauptband, S. 247

28. 11.1960 (Zweite) Verfassung der Republik Tschad s. Hauptband, S. 247

16. 4. 1962 (Dritte) Verfassung der Republik Tschad, Verfass.-Gesetz Nr. 2 Änderungen:

1) 5.6. 1964 Beschluß der Nationalversammlung, ändert Art. 50 (nicht verkündet)

2) 29. 12. 1965 Verfass.-Gesetz Nr. 22, ändert Art. 65, hebt auf Art. 4 (J. O. v. 1. 1. 1966, S. 5)

3) 7. 2. 1967 Verfass.-Gesetz Nr. 7, ändert Art. 13, 59, 61, 64, 65, 76, 77, hebt auf Art. 60, 62, 66, 68 (J. O. v. 15. 6., S. 225) AMTL. TEXT: J. O. Nr. 11 v. 23. 5. 1962, S. 341

ENGL. UBERS.: Peaslee ${ }^{3}$, Africa, S. 65; CAS, S. 167 (Fass. 1965); Peaslee 4 , Africa, S. 105; CCW, Chad (1974)

FRANZ. TEXT: Lavroff, Suppl., S. 48; RJP 1962, S. 267 (Änd. Nr. 1: 1965, S. 625; Nr. 3: 1962, S. 332); NE Nr. 2995 v. 30. 5. 1963, S. 48; Nr. 3411 v. 18. 7. 1967, S. 55 (Fass. 1967) ITAL. ÜBERS.: Foderaro Bd. 1, S. 131 RUSS. ÜBERS.: KGA Bd. 3, S. 644

14. 4. 1975 Außerkraftsetzung der Verfassung durch Militärputsch Einsetzung eines „Cour Suprême Militaire“ am 16. 4.75 


\section{TUNESIEN}

Das franz. Protektorat des Bey von Tunesien wurde am 20. 3. 1956 unabhängig und am 25. 7. 1957 Republik.

25. 7.1957 Entschließung der Verfassungsgebenden Nationalversammlung über die Proklamation der Republik

Dies war die vorläufige Verfassung

ENGL. UBERS.: CCW, Tunisia 1972

1. 6.1959 Verfassung der Republik

ITAL. ÜBERS.: Foderaro, Bd. 3, S. 267

Änderungen:

1) 1. 7.1965, Gesetz Nr. 23, ändert Art. 29 (J. O. v. 2. 7. 1965, S. 825)

2) 30. 12.1969, Gesetz Nr. 63, ändert Art. 51 (J. O. v. 31. 12., S. 1500; RJP 1970, S. 332)

3) 19. 3. 1975 Verfassungsgesetz Nr. 13

ändert Art. 40 u. 51 (J. O.v. 18./21. 3. 1975, S. 520)

AMTL. TEXT: J. O. Nr. 30 v. 1. 6. 1959

DT. UBERS.: Afrika Inf.-dienst v. 15. 6. 1959, Dok. 42

ENGL. UBBERS.: CP Inf. 1959, S. 207 (Änderung Nr. 1:1966, S. 187); YHR 1959, S. 283 (Auszug); State Papers Bd. 164, S. 314; Peaslee $^{3}$, Africa, S. 909; CAS, S. 1578; CCW, Tunisia 1972 (Fass. 1965); Peaslee ${ }^{4}$, Africa, S. 990

FRANZ. TEXT: Inf. CP 1959, S. 204 (Änderung Nr. 1:1966, S. 187); NE Nr. 2633 v. 20. 2. 1960

ITAL. UBERS.: O. M. 1959, S. 411; RI 1959, S. 1027; Rivista di studi politici int. 1959, S. 309; Foderaro Bd. 3, S. 267 (Fass. 1965)

RUSS. UBBERS.: KGA Bd. 3, S. 455

SPAN. UBERS.: BLE 1959, Bd. 26, S. 439

28.11.1966 Gesetz Nr. 67 betr. Errichtung eines Rates der Republik

AMTL. TEXT: J. O. Nr. 51 v. 29. 11./2. 12. 1966, S. 1672

7.11. 1969 Dekret Nr. 400 betr. Schaffung eines Premierministers

AMTL. TEXT: J. O. Nr. 46 v. 7. 11. 1969, S. 1295

FRANZ. TEXT: RTD 1969/70, S. 93

\section{UGANDA}

Am 9. 10. 1962 als Föderation unabhängig. Am 9. 10. 1963 Republik. Am 15. 4. 1966 werden die Rechte der Gliedstaaten weitgehend eingeschränkt und am 8. 9. 1967 in einer unitarischen Verfassung endgültig beseitigt.

Vorschriften bis 1962, Hauptband S. 253.

1962

Uganda (Constitution) Order in Council

Constitution of Uganda (Schedule 2)

Änderungen:

1) 9. 10.1963 (A. 61/63) Republik

2) 7. 10. 1964 (A. 30/64) ändert S. 8 der Order in Council 
15. 4.1966

8. 9.1967

2. 2. 1971

2. 2. 1971

2. 2. 1971

13. 3. 1971
3) 29.12.1964 (A. 36/64) ändert die Grenze von Buganda zugunsten Busoga

AMTL. TEXT: S. I. 2175; Laws of Uganda 1964 I, S. 12

The Constitution of Uganda

Die Verfassung von Ankole, Buganda, Bungoro, Toro und Busoga sind Schedule 1-5

Ainderungen:

1) 28. 5. 1966 (A. 9/66)

AMTL. TEXT: Laws of Uganda 1966 (ohne Nr.)

Constitution of the Republic of Uganda

Änderungen:

1) 7. 5. 1970 (A. 2/70) ändert XIII/1

AMTL. TEXT: Nicht als Act veröffentlicht

Proklamation der Machtübernahme durch General Amin

(Suspendiert c. IV und V der Verfassung: überträgt alle Staatsgewalt dem Military Head of State)

AMTL. TEXT: L. N. 1/71

ENGL. TEXT: CCW (1973)

Establishment of a Defence Council Decree

AMTL. TEXT: D. 1/71

ENGL. TEXT: CCW (1973)

Local Administration Urban Authorities Decree

AMTL. TEXT: D. 2/71

ENGL. TEXT: CCW (1973)

Constitution (Modification) Decree

(ändert den Titel des Staatoberhauptes in Präsident)

AMTL. TEXT: D. 5/71

ENGL. TEXT: CCW (1973)

\section{ZAIRE}

Unabhängig von Belgien am 30. 6. 1960; seit 27. 10. 1971 Ersetzung von „Kongo“ durch „Zaire“.

19. 5.1960 Grundgesetz über die Struktur des Kongo (Erste Verfassung) außer Kraft: 30. 9. 1963

Änderungen:

1) 16. 6. 1960, ändert Art. 110 u. 114 (M. B. v. 24. 6., S. 4780)

2) 9. 3. 1962, ändert Art. 7 (Moniteur v. 5. 4., S. 92)

3) 4. 7. 1963, Gesetz, ändert Art. 247 u. 248 (Moniteur v. 15. 7., S. 123)

4) 18. 7. 1963, Verfassungsgesetz, ändert Art. 40, 41, hebt auf Art. 189, 190, 253, 254 (Moniteur v. 19. 5., S. 423)

AMTL. TEXT: M. B. v. 27./28. 5. 1960, S. 3988;

Moniteur (Congolais) Nr. 21 bis v. 27. 5. 1960, S. 119

ENGL. ÜBERS.: YHR 1960, S. 66 (Auszug)

FRANZ. TEXT: CPE 1960, S. 595 (Änd. S. 625);

RJP 1962, S. 285; Perin, S. 115 (als Entwurf)

RUSS. UBERS.: KGA Bd. 1, S. 258 
17. 6.1960

29. 9.1960

9. 2. 1961

1. 8.1964

7. 3.1966

21. 10. 1966

24. 6.1967

23. 12.1970

ANHANG:

11. 7.1960

4. 8.1960

24. 6. 1961
Grundgesetz betr. Freiheitsrechte

AMTL. TEXT: M. B. v. 24. 6. 60, S. 4781; Moniteur (Congolais) v. 27.6.1960, S. 1916

RUSS. ÜBERS.: KGA, Bd. 1, S. 314

Verfassungsdekretgesetz

s. Hauptband S. 139

Verfassungsdekretgesetz betr. Legislative u. Executive

AMTL. TEXT: Moniteur Nr. 5 v. 9. 2. 1961, S. 39

FRANZ. TEXT: CPE 1961, S. 1070

(Zweite) Verfassung

in Kraft: 1. 8. 1964-14.7. 1967

AMTL. TEXT: Moniteur v. 1. 8. 1964, No. spécial, S. 1

ENGL. UBBERS.: Rev. égypt. 1964, Dok., S. 113, Peaslee 3 , Africa S. 102; Brinton, Constitution of the Congo, Washington 1964

FRANZ. TEXT: RJP 1964, S. 611; JÖR 1966 (Bd. 15), S. 665

Ordonnance-loi Nr. 92 bis: Präsident übernimmt Legislative

AMTL. TEXT: Moniteur v. 15. 4. 1966, S. 158

Ordonnance-loi Nr. 621: Wiedereinsetzung des Parlaments

AMTL. TEXT: Moniteur Nr. 1 v. 1. 1. 1967, S. 1

(Dritte) Verfassung der demokrat. Republik Kongo

Unterzeichnung: 3. 4. 1967

in $\mathrm{Kraft}:$ 15. 7. 1967

Änderungen:

1) 23. 12. 1970, Verfassungsänderungsgesetz Nr. 001 : ändert Art.

4, 19, 21, 22, 37, 39, fügt ein Art. 19 bis (Moniteur v. 1. 1. 1971.

S. 5; VRÜ Beilage zur Nr. 2/72, S. 12)

2) 29. 10. 1971, Verfassungsänderungsgesetz Nr. 006: ändert Art. 1 II sowie ersetzt überall Kongo durch Zaire (Moniteur v. 1. 11.1971 ; a. a. O., S. 15)

AMTL. TEXT: Moniteur Nr. 14 v. 15. 7. 1967, S. 564

DT. UBBERS.: Die Verfassung der Demokrat. Republik Kongo, Köln 1970, S. 26 (S. 27 französ.)

ENGL. ÜBERS.: YHR 1967, S. 73 (Auszug); CCW, Congo (Kinshasa), 1971; Crabb, The Legal System of Congo-Kinshasa, Charlottesville 1970, S. 147; Peaslee ${ }^{4}$, Africa, S. 1028

FRANZ. TEXT: RJP 1969, S. 190

ITAL. ÜBERS.: Foderaro Bd. 1, S. 163

Gesetz Nr. 002: Präsident übernimmt wieder Legislative

AMTL. TEXT: Moniteur Nr. 1 v. 1. 1. 1971, S. 6

\section{NDERGEBIETE}

Unabhängigkeitserklärung der Republik Katanga

ENGL. TEXT: State Papers Bd. 164, S. 669

Verfassung der Republik Katanga

in Kraft: 5. 8. 1959

AMTL. TEXT: Moniteur Katangais Nr. 1 v. 8. 8. 1960, S. 1

FRANZ. TEXT: CPE 1960, S. 762

Übereinkommen Katangas mit Kongo (Léopoldville)

ENGL. TEXT: State Papers Bd. 166, S. 450 


\section{ZAMBIA}

Das britische Protektorat Nord-Rhodesien, bis 1963 Teil der Föderation von Rhodesien und Nyasaland wurde am 24. 10. 1964 als Republik Zambia unabhängig. Vorschriften bis 1962, Hauptband S. 315. 1962

20. 12.1963

10. 8.1964

24. 10.1964

25. 8.1973
Northern Rhodesia (Constitution) Order in Council AMTL. TEXT: S. I. 1814/62; G. N. 213/62

Northern Rhodesia (Constitution) Order in Council Anderungen:

1) 23. 6.1964 (S. I. 919)

2) 27. 7.1964 (S. I. 1191)

AMTL. TEXT: S. I. 2088; G. N. 25/63

Zambia (Election of First President) Act

AMTL. TEXT: S. I. 1783

Zambia Independence Order in Council. Constitution of Zambia Änderungen:

1) 1965 (A. 42/65), ändert S. 125

2) 27.7 .1966 (A. 30/66), ändert $S .12$

3) 18.9 .1966 (A. 47/66), ändert S. 44, 65

4) 28.6. 1967 (A. 33/67), ändert S. 125

5) 1968 (A. $1 / 68)$

6) 1968 (A. $2 / 68)$

7) 11. 2. 1969 (A. 1/69), ändert S. 47, 56, 115, 125

8) 13.3 .1969 (A. 2/69), neuer S. $113 \mathrm{~A}$

9) 24. 4.1969 (A. $10 / 69$, ändert S. 72

10) 21. 10. 1969 (A. 32/69, ändert S. 62

11) 21. 10. 1969 (A. 33/69), ändert S. 10, 18, 24, 26, 29, 44, $62,65,99$ neue S. 26 A, 109 A

12) 28. 1. 1970 (A. 12/70), ändert S. 110, 113, neue S. 112 A

13) 5. 10.1970 (A. 43/70), ändert S. 125

14) 14.10 .1970 (A. 44/70), ändert S. 49

15) 19. 12. 1970 (A. 58/70), ändert S. $18,44,65,96,97,99$, 100

16) 23. 3. 1972 (A. $1 / 72$ ), ändert S. $52,98,105,125$

17) 18.8 .1972 (A. 17/72), neue S. $109 \mathrm{C}$

18) 13. 12. 1972 (A. 27/72), ändert S. 110

19) 13. 12.1972 (A. 28/72), ändert S. 107

20) 15. 12. 1972 (A. 29/72), neues Ch II A (Einparteienstaat) AMTL. TEXT: S. I. 1652

ENGL. TEXT: Peaslee ${ }^{3}$ I, S. 1027; Peaslee ${ }^{4}$, Africa, S. 1051 (Fass. 1970)

Constitution of Zambia

AMTL. TEXT: A. 27/73

ENGL. TEXT: VRU, Verfassungstexte, Heft 1/1974 


\section{ZENTRALAFRIKA}

Das frühere Ubangi-Schari wurde unabhängig von Frankreich am 13. 8. 1960 als Zentralafrikanische Republik.

16. 2. 1959 Verfassung der ZAR, Gesetz Nr. 9 (in der Communauté) s. Hauptband S. 268

14. 8. 1960 Gesetz Nr. 156: Der Präsident der Regierung wird Staatspräsident

AMTL. TEXT: J. O. v. 1. 10.60

12.12. 1960 Gesetz Nr. 163 über die Änd. der Verfassung der ZAR

Von der Verfassung v. 1959 wurden aufgehoben Art. 9, 1618, 43-44; geändert Art. 1, 2, 5-8, 11-14, 19-21, 31, 32, 34, 35, 37-39, 42; ferner wurden überall eingesetzt: „Assemblée National" und Président de la République“.

Beschluß: 17. 11. 1960

Promulgation: 12. 12. 1960 (Dekret)

Anderungen:

1) 4. 5. 1961, Gesetz Nr. 220 (Beschluß v. 20. 4.), ändert die Nummern, indem die 6 aufgehobenen Artikelnummern aufrückten (J. O. v. 15. 5., S. 128)

2) 28. 12. 1962, Gesetz Nr. 365, ändert Art. 2, 5, 12, (J. O. v. 15. 1. 1963, S. 31)

3) 19. 11. 1963, Gesetz Nr. 426, ändert Art. 10 (J. O. v. 1. 12., S. 600)

AMTL. TEXT: J. O. v. 15. 12. 1960, S. 663

ENGL. UBBERS.: Peaslee ${ }^{3}$, Africa, S. 50 (Fass. 1962)

FRANZ. TEXT: Lavroff, S. 224; Muracciole, S. 13; RJP 1961, S. 13; RJP 1961, S. 236; NE Nr. 2995 v. 27. 5. 1963, S. 26;

Nr. 3175 v. 26. 3. 1965 , S. 25 (Änd. Nr. 1-2)

ITAL. UBBERS.: Foderaro Bd. 1, S. 119 (Fass. 1962)

26. 11. $1964 \quad$ Verfassung der Republik, Gesetz Nr. 37

AMTL. TEXT: J. O. v. 1. 1. 1965, S. 23

FRANZ. TEXT: RJP 1965, S. 149

RUSS. UBERS.: KGA Bd. 3, S. 620

4. 1.1966 Verfassungsakt Nr. 1: Aufhebung der Verfassung v. 1964

AMTL. TEXT: J. O. v. 15. 1. 1966, S. 19

FRANZ. TEXT: RJP 1966, S. 361; CCW C. A. R., 1972, S. 1;

NE Nr. $3833 / 34$ v. 10.11 .1971 , S. 67

8. 1. 1966 Verfassungsakt Nr. 2: Vorläufige Verfassung

AMTL. TEXT: J. O. v. 15. 1. 1966, S. 20

FRANZ. TEXT: RJP 1966, S. 361; CCW C. A. R., 1972, S. 2

$\mathrm{NE}$ a. a. O., S. 7 